\title{
Mechanics of generically creased disks
}

\author{
M. G. Walker $\oplus^{*}$ \\ Department of Engineering Science, University of Oxford, Parks Road, Oxford OX1 3PJ, United Kingdom
}

(Received 6 December 2019; revised manuscript received 17 February 2020; accepted 20 March 2020; published 16 April 2020)

\begin{abstract}
Folded structures are often idealized as a series of rigid faces connected by creases acting as revolute hinges. However, real folded structures can deform between creases. An example of particular interest is a disk decorated by multiple radial creases. Such disks are bistable, snapping between a "natural" and "inverted" shape. We investigate the mechanical behavior of these creased disks and propose a new analytical approach to describe their mechanics. Detailed experiments are performed which show that, when indented at the center, a localized dimple forms, precluding the conical shape assumed in previous studies. As the indentation depth increases this dimple expands radially until reaching the disk edge when it snaps to the inverted shape, which has a conical form. We develop an analytical model which approximates each face as a series of rigid facets connected by hinges that can both rotate and stretch. Energy expressions are derived relating hinge rotation and stretching to compatible shell deformations of the facets and equilibrium enforced by minimizing the total strain energy. By increasing the number of facets, the mechanics of the continuum shell is approached asymptotically. The analysis shows that membrane stretching of the faces is required when a conical form of deformation is enforced. However, in the limit of zero thickness, the forming and propagation of a localized dimple is inextensional. This new approach relates the kinematic analysis of rigid origami to the mechanics of thin shells, offering an efficient method to predict the behavior of folded structures.
\end{abstract}

DOI: 10.1103/PhysRevE.101.043001

\section{INTRODUCTION}

Crease patterns observed in nature, or formed by phenomena such as crumpling, have inspired origami designs with applications ranging from deployable aerospace structures $[1,2]$ to mechanical metamaterials [3,4]. Analysis usually assumes rigid faces connected by creases acting as revolute hinges, with deformation being purely kinematic. The addition of torsional stiffness to the hinges provides global mechanical behavior but does not influence the underlying kinematics. Such "rigid origami" imposes strict constraints on the crease layout [5] and accessible geometric configurations.

Structures made from folded thin sheets can deform between the creases, introducing additional soft modes of deformation [6]. This can be accounted for using finite element analysis $[7,8]$, or sometimes analytically by assuming inextensibility. However, reduced order models offer a more computationally efficient method. Examples include additional diagonal bending lines within the rigid faces [9] or hinges along diagonal bars in bar-and-hinge models [6,10-12]. These methods allow some flexibility of faces within the context of rigid origami, but they do not capture the full continuum shell deformations, in particular, membrane stretching.

The extent to which deformation of a folded sheet is governed by crease opening or face bending is determined by an origami length scale, $L^{*} \equiv D / k$, where $D$ is the shell flexural rigidity of the face and $k$ is the crease torsional stiffness [13]. When the characteristic size of a face $l \gg L^{*}$,

\footnotetext{
*martin.walker@some.ox.ac.uk
}

behavior is dominated by face bending. In this case, the creases provide boundary constraints for the continuum shell deformation of the faces between them. This offers the possibility of interesting new mechanical behavior at the cost of considerably increased analytical complexity. An example of particular interest is the bistable behavior of a thin disk decorated by multiple radial creases, as shown in Fig. 1. This behavior is observed by folding a piece of paper, then while supporting the paper at the edges of the crease, pressing downward at a point along the crease line. After some initial elastic resistance, a snap-through occurs leaving the crease with a sharp localized vertex. Except for this vertex the creases are otherwise straight.

Lechenault and Adda-Bedia [14] investigate the shape of these creased disks by assuming each face between creases deforms as a conical surface, with generators extending radially from a central vertex, forming a foldable cone or "f-cone." Assuming inextensibility, they identify the metastable states corresponding to configurations of minimum bending energy. Andrade-Silva et al. [15] build on this analysis by incorporating geometric nonlinearity but retaining the inextensibility assumption. They also include the influence of the crease opening stiffness and find that when it is very stiff face stretching becomes important to the mechanical behavior. Walker and Seffen [16] consider the similar deformation of a creased strip bent perpendicular to the crease axis. They approximate each face of the strip as a set of rigid facets connected by rotational hinges emanating from a central vertex. Using a Gauss mapping approach they establish the kinematic relationship between facets. An energy cost is assigned by relating hinge rotations to compatible shell deformation of the adjacent facet. 


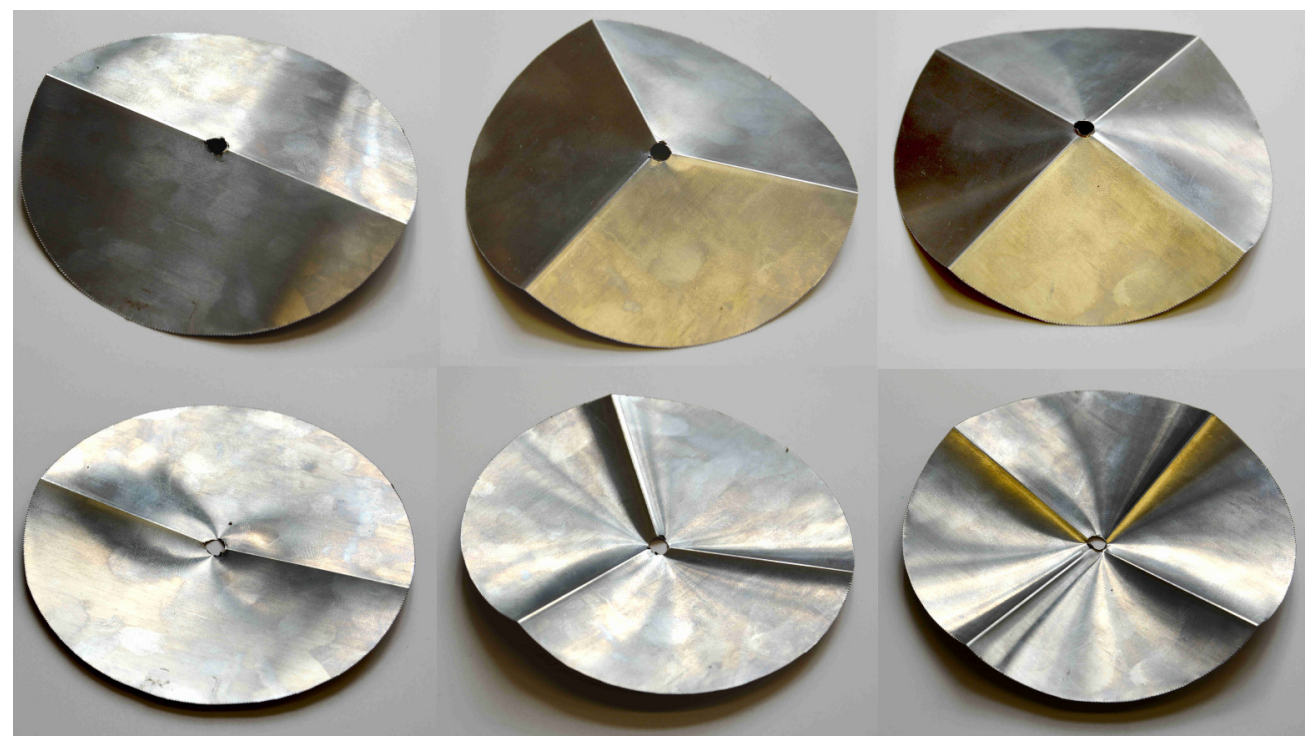

FIG. 1. Disks with 2, 3, and 4 radial creases in the natural (top) and inverted (bottom) configuration.

By increasing the number of facets, the continuum solution of [14] is approached asymptotically. All of these approaches assume inextensible deformation with straight generators, or hinges, originating from a single central vertex. This precludes any bending along their length, including along the crease line. Additionally, due to the constraint provided by the presence of stiff creases, a complete mechanical description must include membrane stretching, which is not possible using current approaches.

In this study we propose a new analysis technique for creased shells that includes both bending and membrane stretching deformations. In particular, we consider the mechanics of a thin disk decorated by multiple radial creases. We perform careful experiments using thin folded steel disks indented at the center and show that deformation does not conform to the conical assumption made in previous studies. Instead, a localized dimple forms around the indentation point, in a manner similar to the indentation of curved thin shells [17]. Under increasing deformation this dimple expands radially. When it reaches the edge, the disk snaps to the inverted shape.

To capture this behavior we divide each face between creases into a series of rigid facets, which extend from the central vertex to the outer edge of the disk, and are connected by deformable hinges. By allowing these hinges to both rotate and stretch, we obtain the complete mechanical behavior corresponding to the conical deformation assumed in previous studies. However, this eliminates the possibility of crease bending and the forming of a localized dimple. Therefore, we modify this model to allow for bending of the creases, and facets, to capture the localized deformation. When combined these two models show good agreement with experiments.

We proceed as follows: first, we discuss experiments conducted using folded steel disks. This is followed by the derivation of a rigid-faceted model that assumes the creases remain straight and facets remain flat. This model is then modified to allow for bending of the creases and facets. The models are then compared to experiments and we conclude.

\section{EXPERIMENTS}

Tests were performed using $100 \mathrm{~mm}$ diameter disks cut from 0.10-mm-thick stainless steel sheet (AISI 301). To avoid the influence of a stress singularity at the vertex, all disks had a central hole with a diameter of $3 \mathrm{~mm}$. Radial creases were formed by pressing the disks against a stiff rubber sheet using a die press. For simplicity, experiments were limited to disks with two or three equally spaced radial creases. Each disk was $3 \mathrm{D}$ scanned to obtain the initial fold angle across the crease, $\beta$ [see Fig. 2(a)]. In the case of a diametrical crease, a best-fit plane was found for the face on each side of the crease and the angle between their normals used as the fold angle. In the case of three radial creases, the scanned shape was cut by a plane perpendicular to each crease line at its midpoint. A best fit curve was obtained for each side of the crosssection and the angle at each intersection measured. The average of these angles is used as the crease angle for the disk.

During testing the disks were supported along the crease line by cone-pointed bolts located $40 \mathrm{~mm}$ from the center. Since the diametrically creased disk is supported at only two points it is inherently unstable. Therefore, at each end of the crease, stabilizing pins were attached which fit into a slot in the test fixture to stabilise the disk during testing, as shown in Fig. 2(b). The disks were loaded using an indenter with a 4-mm-diameter point. From this point a 2-mm-diameter smooth rod is attached and passed through the central hole in the disk. This improves the stability of the test by preventing the disk from sliding off the supports. The three-crease disks were supported along the creases by three cone-pointed bolts, located $40 \mathrm{~mm}$ from the center. Stabilizing pins were not needed at the end of the creases since this geometry is stable. The three-crease disks were loaded with the same indenter and stabilizing pin as the diametrically creased disks. Tests were conducted using an Instron universal testing machine (model $5582)$ with a $100 \mathrm{~N}$ Class $1( \pm 1.0 \%)$ load cell. Each disk was tested three times under displacement control at a rate of 5 $\mathrm{mm} / \mathrm{min}$. 


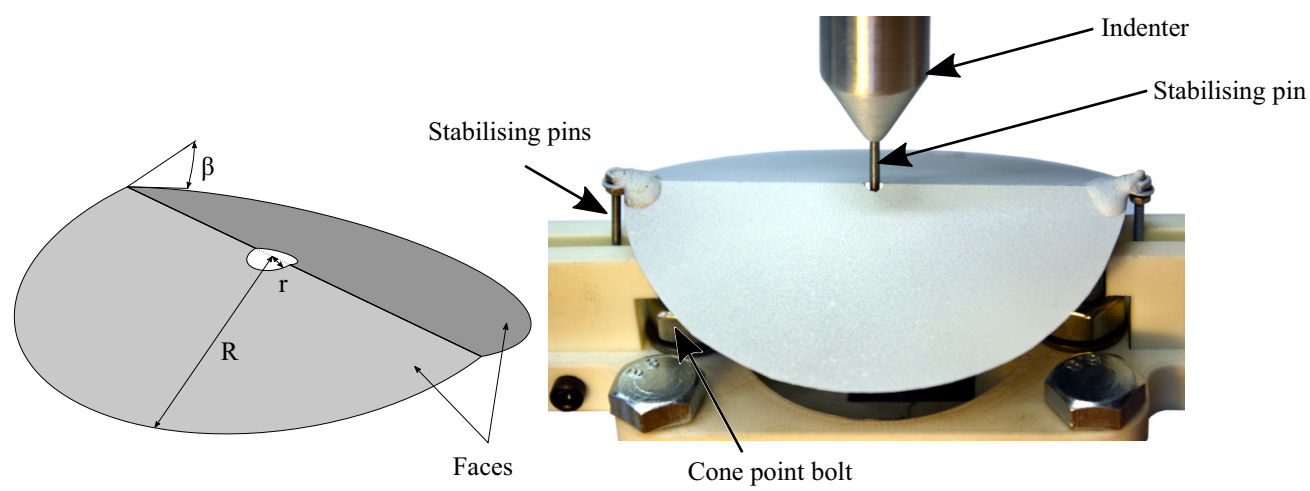

(a)

(b)

FIG. 2. A schematic drawing of a diametrically creased disk (a) with a fold angle of $\beta$, disk radius $R$ and hole radius $r$. A creased disk is shown mounted in the test fixture in (b). Stabilizing pins are located at the outer edges of the crease and fit into a slot in the test fixture. An additional stabilizing pin extends from the tip of the indenter and passes through the central hole. The disk is balanced on two cone pointed bolts positioned beneath the crease.

The indentation force-displacement results are shown in Fig. 3. Initially a localized dimple forms surrounding the indenter, as shown in Fig. 4. Under increasing indentation, the dimple region grows in size with the ridge moving outward toward the disk edge. Cross-sections showing the outward movement of the ridge are shown in Fig. 5. The rate of dimple growth appears to increase as the ridge nears the disk edge. When the ridge reaches the outer edge, the disk snaps to the inverted shape. The snap-through separates the disk from the indenter, and zero reaction force is measured. Eventually the indenter reaches the disk in its inverted state and the reaction force increases approximately linearly.

The peak reaction force increases as the crease angle, $\beta$, is increased and the displacement at snap-through also increases. A similar process is observed with the three radial crease disks, as shown in Fig. 3(b). However, the snap-through is approached more smoothly and the sharp drop in load observed for the diametrically creased disks is not present. We proceed to derive an analytical model to capture the observed behavior.

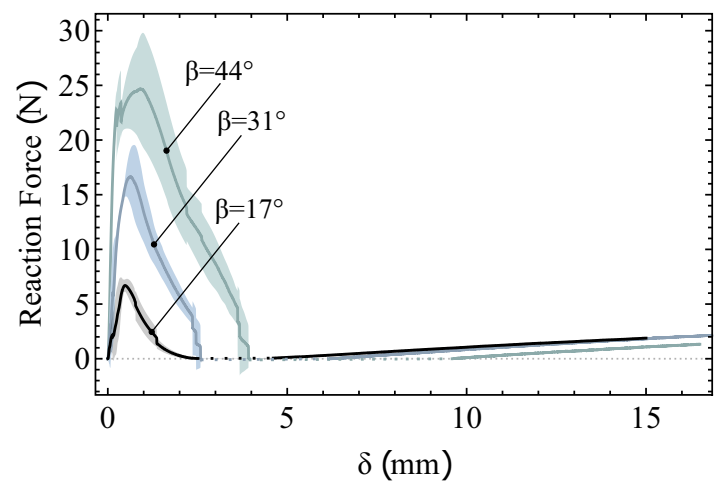

(a)

\section{ANALYSIS}

\section{A. Straight crease}

We first assume that hinges emanating from the central vertex remain straight. While this precludes the localized dimple observed in experiments, it corresponds to the assumptions of previous studies on the inversion of $\mathrm{f}$-cones $[14,15]$. We therefore find the consequences of this assumption on the predicted mechanical behavior. For conciseness the analysis is restricted to symmetric behavior, such that all creases deform simultaneously and identically. However, the analysis can be adapted for general deformations.

Consider an initially planar thin disk with thickness $t$, radius $R$, and a small hole of radius $r$ located at the center, as shown in Fig. 6. $N$ equally spaced radial creases are imposed dividing the disk into $N$ identical faces. Therefore, only one face, of angle $2 \pi / N$, is considered for this analysis. This face is divided by $2 n$ equally spaced radial hinges, forming $2 n+1$ wedge-shaped facets, each subtending an angle of $\alpha=$ $2 \pi / N(2 n+1)$. Mirror symmetry is assumed across the face bisector and only one half is analyzed, shown shaded in Fig. 6. The facets are all joined at the central vertex but are otherwise

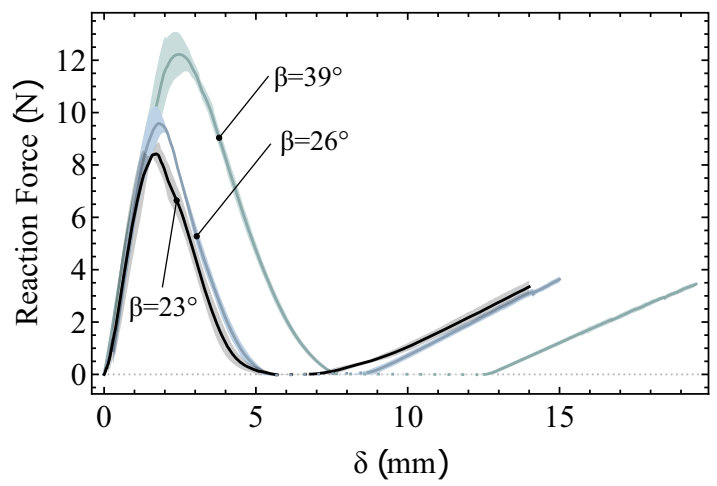

(b)

FIG. 3. Experimental results for disks with (a) two and (b) three radial creases. The solid line is the mean of three tests and the shaded region shows one standard deviation from the mean. Dotted lines indicate separation of the indenter from the disk. 


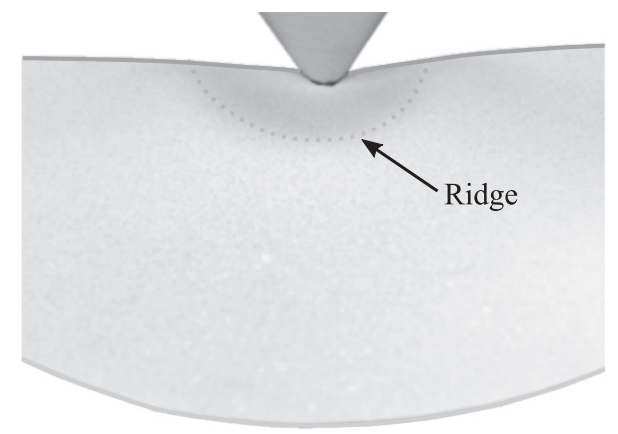

FIG. 4. Initially a localized dimple forms at the indentation point. Under increasing indentation the dimple grows and the ridge moves toward the outer edge. Once the ridge reaches the outer edge, the disk snaps to the inverted shape.

unconstrained. We number the facets clockwise, with facet $i$ located between hinges $i-1$ and $i$. The centerline of facet $n+1$ is aligned with the line of symmetry. A Cartesian coordinate system is located at the center of the undeformed disk with the $y$ axis aligned radially along a crease and the $z$ axis pointing out of the page. Initially, the facets on each side of a crease are rotated about the crease line by an angle of $\beta / 2$ from the $x-y$ plane. This leaves an angle $\beta$ across each crease line, with the apex oriented in the positive $z$ direction (i.e., mountain folds), as shown in Fig. 7. We assume the radius, $R$, is sufficiently large such that the crease angle, $\beta$, is effectively fixed, forming a boundary condition on the deformation of the adjoining faces [13].

The facets must separate and move from the planar state to accommodate the imposed crease angle. We allow the hinges to both rotate and stretch and impose these hinge deformations as boundary conditions on the subsequent facet, then derive the energy cost of the corresponding continuum shell deformations. The equilibrium shape is thus obtained by minimizing the total strain energy with respect to the hinge rotations and stretch angle. The analysis proceeds by incrementally moving the center of the disk down/upward, causing the creases to form an angle $\Psi$ relative to the $x-y$ plane, as shown in Fig. 7. At each deformation increment, the equilibrium shape and strain energy are obtained. We first consider the kinematics of this deformation before deriving the energy cost.

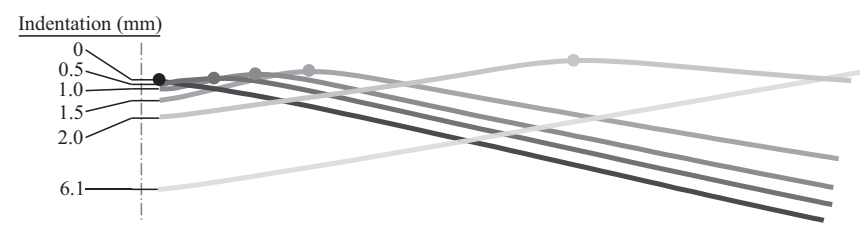

FIG. 5. Radial cross-sections from 3D scans of a diametrically creased disk taken during testing. The cross-sections are taken from the center of the disk perpendicular to the crease. The peak of the ridge is indicated by the filled circles. Under increasing indentation the ridge moves outward toward the disk edge.

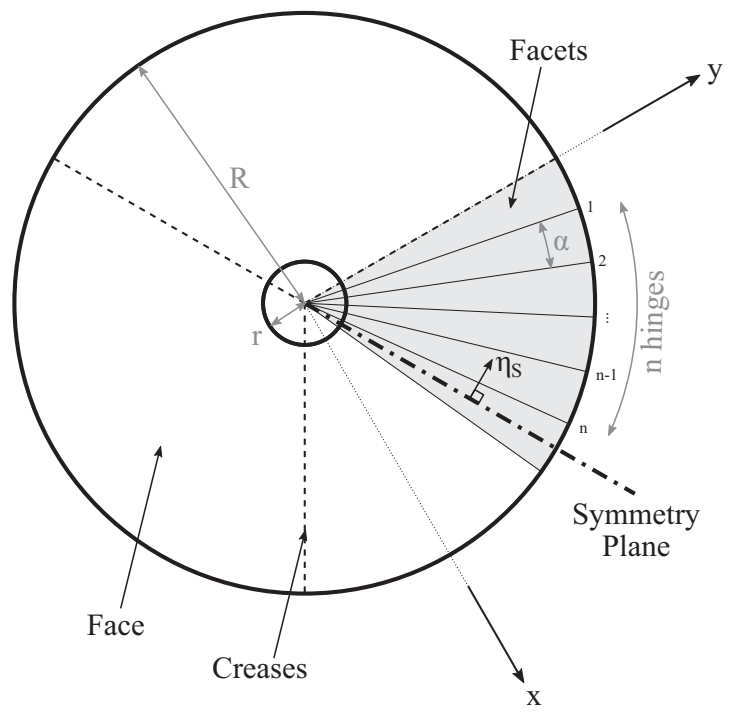

FIG. 6. Plan view schematic of a disk with radius $R$ and a small hole of radius $r$ located at the center. The three radial creases are shown dashed. Each face between two creases is subdivided into $2 n+1$ facets which each subtend an angle $\alpha$. Mirror symmetry is assumed across the face bisector and only one half of a face, shown shaded, is analyzed.

\section{Kinematics}

The kinematics are described by writing the position of facet $i+1$ relative to facet $i$, forming a kinematic chain. We label the unit normal vector of facet $i: \eta_{i}$; and unit vectors along the facet edges: $\boldsymbol{p}_{i, 1}$ and $\boldsymbol{p}_{i, 2}$, as shown in Fig. 7. The normal and edge vectors of the first facet, adjacent to the crease, are set by the crease angle, $\beta$, the facet angle, $\alpha$, and the imposed deformation angle, $\Psi$ :

$$
\begin{gathered}
\boldsymbol{\eta}_{1}=\left[\begin{array}{c}
\sin \frac{\beta}{2} \\
-\cos \frac{\beta}{2} \sin \Psi \\
\cos \frac{\beta}{2} \cos \Psi
\end{array}\right] \quad \boldsymbol{p}_{1,1}=\left[\begin{array}{c}
0 \\
\cos \Psi \\
\sin \Psi
\end{array}\right] \\
\boldsymbol{p}_{1,2}=\left[\begin{array}{c}
\sin \alpha \cos \frac{\beta}{2} \\
\sin \alpha \sin \frac{\beta}{2} \sin \Psi+\cos \alpha \cos \Psi \\
\cos \alpha \sin \Psi-\sin \alpha \sin \frac{\beta}{2} \cos \Psi
\end{array}\right] .
\end{gathered}
$$

Facet $i+1$ is initially aligned in the same plane as facet $i$, then rotated by an angle $\theta_{i}$ about the vector $\boldsymbol{p}_{i, 2}$. Facet $i+1$ is then rotated about its normal vector $\left(\boldsymbol{\eta}_{i+1}\right)$ by an angle $\phi$, as shown in Fig. 7. The stretch angle, $\phi$, is assumed to be constant for all facets, which implies constant circumferential strain. Making use of Rodrigues' rotation formula, the orientation of facet $i+1$ is expressed relative to facet $i$ as

$$
\begin{aligned}
\boldsymbol{\eta}_{i+1}= & \boldsymbol{\eta}_{i} \cos \theta_{i}+\left(\boldsymbol{p}_{i, 2} \times \boldsymbol{\eta}_{i}\right) \sin \theta_{i} \\
& +\boldsymbol{p}_{i, 2}\left(\boldsymbol{p}_{i, 2} \cdot \boldsymbol{\eta}_{i}\right)\left(1-\cos \theta_{i}\right), \\
\boldsymbol{p}_{i+1,1}= & \boldsymbol{p}_{1,2} \cos \phi+\left(\boldsymbol{\eta}_{i+1} \times \boldsymbol{p}_{1,2}\right) \sin \phi \\
& +\boldsymbol{\eta}_{i+1}\left(\boldsymbol{\eta}_{i+1} \cdot \boldsymbol{p}_{1,2}\right)(1-\cos \phi), \\
\boldsymbol{p}_{i+1,2}= & \boldsymbol{p}_{i+1,1} \cos \alpha-\left(\boldsymbol{\eta}_{i+1} \times \boldsymbol{p}_{i+1,1}\right) \sin \alpha \\
& +\boldsymbol{\eta}_{i+1}\left(\boldsymbol{\eta}_{i+1} \cdot \boldsymbol{p}_{i+1,1}\right)(1-\cos \alpha) .
\end{aligned}
$$




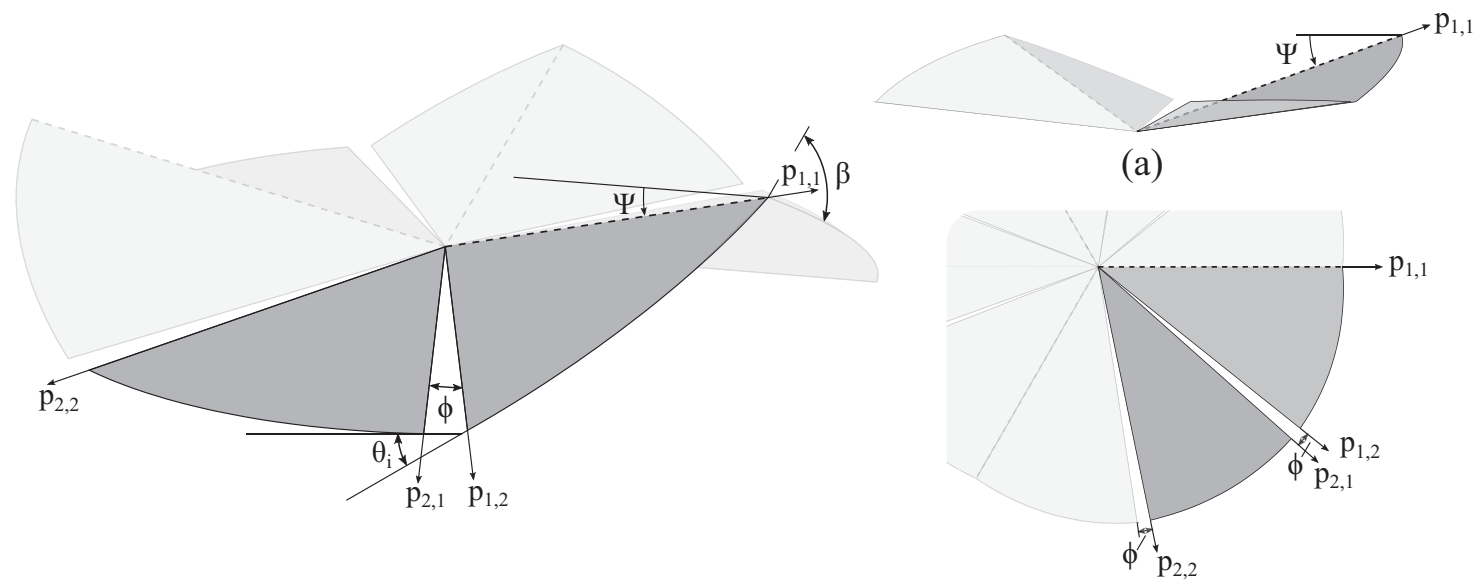

(b)

FIG. 7. Deformed shape of a disk with $N=3$ creases and $n=1$ hinge lines per half-face. A fixed angle, $\beta$, is imposed across each crease line. The facets separate by an angle $\phi$ and the rotation angle between facet $i$ and $i+1$ is $\theta_{i}$. The center of the disk is moved down/upward with all creases forming an angle $\Psi$ to the $x-y$ plane. A side view is shown in (a) and (b) shows a view of along the normal of facet 2 .

The deformation of each face is symmetrical about its bisector. As a result, facet $n+1$ is split by a symmetry plane and can only move vertically during deformation, thus providing a kinematic boundary condition. The unit normal vector of the symmetry plane, which does not change during deformation of the disk, is

$$
\eta_{S}=\left[\begin{array}{lll}
-\cos (\pi / N) & \sin (\pi / N) & 0
\end{array}\right]^{T} .
$$

To enforce the symmetry condition, the normal of the symmetry plane, $\boldsymbol{\eta}_{S}$, must align with the normal vector of the bisecting plane of facet $n+1$. The unit vector along the bisector of facet $n+1$ is

$$
\begin{aligned}
\boldsymbol{b}_{n+1}= & \boldsymbol{p}_{n+1,1} \cos \frac{\alpha}{2}-\left(\boldsymbol{\eta}_{n+1} \times \boldsymbol{p}_{n+1,1}\right) \sin \frac{\alpha}{2} \\
& +\boldsymbol{\eta}_{n+1}\left(\boldsymbol{\eta}_{n+1} \cdot \boldsymbol{p}_{n+1,1}\right)\left(1-\cos \frac{\alpha}{2}\right) .
\end{aligned}
$$

Since the normal of the bisecting plane of facet $n+1$ is: $\boldsymbol{\eta}_{n+1} \times \boldsymbol{b}_{n+1}$, the symmetry boundary condition is obtained by the vector triple product:

$$
\boldsymbol{\eta}_{S} \times\left(\boldsymbol{\eta}_{n+1} \times \boldsymbol{b}_{n+1}\right)=\mathbf{0} .
$$

The equilibrium shape of the creased disk is obtained by minimizing the total strain energy with respect to the hinge deformations, $\theta_{i}$ and $\phi$, subject to the constraint provided by Eq. (5).

\section{Energy}

To compute the deformation energy as a result of the hinge rotations, $\theta_{i}$, and stretch, $\phi$, we use the energy cost of compatible deformations of initially flat facets. The hinge rotation between facet $i$ and $i+1, \theta_{i}$, is split between the edges of the subsequent facet $(i+1)$ resulting in a rotation of $\theta_{i} / 2$ being applied along each edge in the opposite sense, as shown in Fig. 8(a). As a result of these edge rotations the facet forms a conical deformed shape. The strain energy of this deformation for each facet is summed to compute the total bending energy.
The separation angle between facets, $\phi$, is assumed to be the same between all facets. To compute the energy cost of this separation we impose the stretching angle on a flat facet which initially subtends and angle $\alpha$. This causes an increase of the facet subtended angle to $\alpha+\phi$, as shown in Fig. 8(b). The strain energy of this change in subtended angle is summed for all facets to compute the total stretching energy. Bending and stretching deformations are considered separately and we neglect their interaction since this higher order effect is negligible when the number of facets is large.

a. Bending. We consider a facet with subtended angle, $\alpha$, inner radius, $r$, and outer radius, $R$. Edge rotations of $\theta_{i} / 2$ are imposed along each edge, as shown in Fig. 8(a). The facet is assumed to form a segment of a developable cone. Following

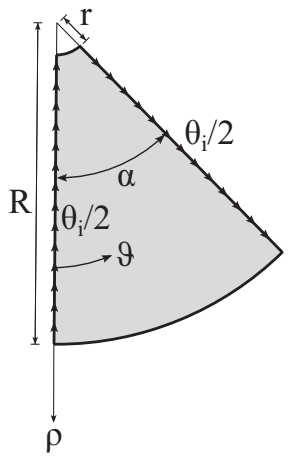

(a)

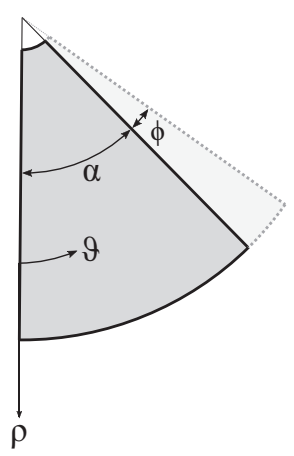

(b)
FIG. 8. The hinge rotations, $\theta_{i}$, and stretch angle between facets, $\phi$, are imposed as boundary conditions of an initially flat facet. The hinge rotations are divided equally between the two edges of each facet such that rotations of $\theta_{i} / 2$ are applied on each edge of facet $i+1$ in the opposite sense, as shown in (a) using the right-hand-rule. The in-plane stretching angle, $\phi$, increases the subtended angle of each facet to $\alpha+\phi$, as shown in (b). To derive the energy cost of these deformations, a cylindrical facet coordinate system is used with $\rho$ as the radial coordinate and $\vartheta$ as the azimuthal coordinate. 
Refs. [14,18], a cylindrical coordinate system is assumed and the deformed shape is written as: $\boldsymbol{\rho}=\rho \hat{\boldsymbol{\rho}}+\rho \Phi(\vartheta) \hat{\boldsymbol{z}}$, where $\rho$ is the radial coordinate, $\vartheta$ is the azimuthal coordinate, and $\hat{z}$ is the facet normal direction $\left(\boldsymbol{\eta}_{i+1}\right)$. The facet bending energy density is therefore

$$
u_{B}=\frac{D}{2} \ln \left(\frac{R}{r}\right) \int_{0}^{\alpha}\left[\Phi^{\prime \prime}(\vartheta)+\Phi(\vartheta)\right]^{2} d \vartheta
$$

where $D=E t^{3} / 12\left(1-v^{2}\right)$ is the shell flexural rigidity, $E$ is Young's modulus, and $v$ is Poisson's ratio. We seek the function $\Psi(\vartheta)$ which minimizes Eq (6). Taking the first variation, the following Euler-Lagrange equation is obtained:

$$
\Phi^{(4)}(\vartheta)+2 \Phi^{\prime \prime}(\vartheta)+\Phi(\vartheta)=0,
$$

which has the solution

$$
\Phi=C_{1} \cos (\vartheta)+C_{2} \vartheta \cos (\vartheta)+C_{3} \sin (\vartheta)+C_{4} \vartheta \sin (\vartheta),
$$

subject to the boundary conditions at the facet edges:

$$
\Phi^{\prime}(0)=-\Phi^{\prime}(\alpha)=\frac{\theta_{i}}{2}, \quad \Phi(0)=\Phi(\alpha)=0 .
$$

Solving for the unknown constants:

$$
\begin{aligned}
& C_{1}=0, \quad C_{2}=\frac{\theta_{i} \sin \alpha}{2(\alpha+\sin \alpha)}, \\
& C_{3}=\frac{\alpha \theta_{i}}{2(\alpha+\sin \alpha)}, \quad C_{4}=-\frac{\theta_{i}(1+\cos \alpha)}{2(\alpha+\sin \alpha)} .
\end{aligned}
$$

By completing the integration in Eq. (6) and summing for all facets, the total bending energy is

$$
U_{B}=D \frac{(1+\cos \alpha)}{2(\alpha+\sin \alpha)} \ln \left(\frac{R}{r}\right) \sum_{i=0}^{n} \theta_{i}^{2} .
$$

As in the case of conical dislocations such as the d-cone $[19,20]$, the bending energy diverges logarithmically as the hole radius is reduced to zero. In reality, localized stretching, yielding, or damage will occur in this region [18].

b. Stretching. The imposed stretching deformation, $\phi$, causes strain in both the azimuthal, $\hat{\vartheta}$, and radial, $\hat{\rho}$, directions [21]:

$$
\epsilon_{\vartheta \vartheta}=\frac{\phi}{\alpha}+\frac{u_{\rho}}{\rho}, \quad \epsilon_{\rho \rho}=\frac{\partial u_{\rho}}{\partial \rho},
$$

where $u_{\rho}$ is the displacement in the radial direction. Since we have assumed $\phi$ is constant, $\epsilon_{\vartheta \vartheta}$ and $\epsilon_{\rho \rho}$ are functions of the radial coordinate, $\rho$, only and are the same for all facets. The stretching energy of each facet is

$$
u_{s}=\frac{E t \alpha}{2\left(1-v^{2}\right)} \int_{r}^{R}\left[\epsilon_{\vartheta \vartheta}^{2}+\epsilon_{\rho \rho}^{2}+2 v \epsilon_{\vartheta \vartheta} \epsilon_{\rho \rho}\right] \rho d \rho .
$$

Taking the first variation we obtain the Euler-Lagrange equation for the radial displacement, $u_{\rho}$, which minimizes Eq. (12):

$$
u_{\rho}^{\prime \prime}-\frac{(1+v)}{\rho^{2}} u_{\rho}=\frac{\phi}{\alpha \rho} .
$$

By solving this differential equation, we obtain the radial displacement,

$$
u_{\rho}=C_{5} \rho^{\frac{1}{2}+\lambda}+C_{6} \rho^{\frac{1}{2}-\lambda}-\frac{\phi}{\alpha(1+v)} \rho,
$$

where $4 \lambda^{2}=4 v+5$. This is subject to the condition of zero radial stress at the inner and outer edges, respectively:

$$
\begin{aligned}
\sigma_{\rho \rho}(r) & =\frac{E t}{1-v^{2}}\left[\epsilon_{r r}(r)+v \epsilon_{\vartheta \vartheta}(r)\right]=0, \\
\sigma_{\rho \rho}(R) & =\frac{E t}{1-v^{2}}\left[\epsilon_{r r}(R)+v \epsilon_{\vartheta \vartheta}(R)\right]=0 .
\end{aligned}
$$

Solving for the unknown constants,

$$
\begin{aligned}
& C_{5}= \frac{2(1-v) r^{\frac{1}{2}-\lambda}\left[\left(\frac{R}{r}\right)^{\lambda+\frac{1}{2}}-1\right]}{(2 \lambda+2 v+1)\left[\left(\frac{R}{r}\right)^{2 \lambda}-1\right]} \frac{\phi}{\alpha}, \\
& C_{6}=\frac{2(1-v) \sqrt{r} R^{\lambda}\left[\sqrt{\frac{R}{r}}-\left(\frac{R}{r}\right)^{\lambda}\right]}{(2 \lambda-2 v-1)\left[\left(\frac{R}{r}\right)^{2 \lambda}-1\right]} \frac{\phi}{\alpha} .
\end{aligned}
$$

The circumferential and radial strains are obtained directly from Eqs. (11):

$$
\begin{gathered}
\epsilon_{\vartheta \vartheta}=\frac{v}{v+1} \frac{\phi}{\alpha}+\frac{1}{\rho^{\lambda+\frac{1}{2}}}\left(C_{5} \rho^{2 \lambda}+C_{6}\right) \\
\epsilon_{\rho \rho}=\frac{-1}{v+1} \frac{\phi}{\alpha}+\frac{1}{2 \rho^{\lambda+\frac{1}{2}}}\left[C_{5}(2 \lambda+1) \rho^{2 \lambda}+(1-2 \lambda) C_{6}\right] .
\end{gathered}
$$

The stretching energy is obtained from Eq. (12), which has a lengthy closed form expression. If $v=5 / 16 \approx 0.31$, then $\lambda=5 / 4$ and Eq. (12) simplifies to

$$
\begin{aligned}
U_{S} \approx & \frac{40 E t}{4851\left(r^{5}+R^{5}-2(r R)^{5 / 2}\right)} \frac{n \phi^{2}}{\alpha} \\
& \times\left[9\left(R^{7}-r^{7}\right)-420\left(\sqrt{r^{9} R^{5}}+\sqrt{r^{5} R^{9}}\right)\right. \\
& +658\left(r^{17 / 4} R^{11 / 4}-r^{11 / 4} R^{17 / 4}\right) \\
& \left.+438\left(r^{21 / 4} R^{7 / 4}-r^{7 / 4} R^{21 / 4}\right)+539\left(r^{2} R^{5}-r^{5} R^{2}\right)\right] .
\end{aligned}
$$

The total strain energy of the entire disk is thus the sum of the bending and stretching energy of every facet:

$$
U_{T}=2 N\left(U_{S}+U_{B}\right) \text {. }
$$

The equilibrium shape of the creased disk is obtained by minimizing Eq. (21) with respect to the rotations, $\theta_{i}$, and stretch, $\phi$, subject to the kinematic constraint given by Eq. (5).

\section{Mechanical behaviour}

To obtain the mechanical behavior of a creased disk we begin with all creases lying in the $x-y$ plane $(\Psi=0)$. The hinge rotations, $\theta_{i}$, and circumferential stretch angle, $\phi$, corresponding to this configuration are obtained by minimizing the total strain energy, Eq. (21), subject to the symmetry constraint [Eq. (5)], using a constrained numerical minimisation scheme in MATLAB [22]. The crease rotation angle, $\Psi$, is then incremented by a small angle, $d \Psi$. Using the hinge rotations 


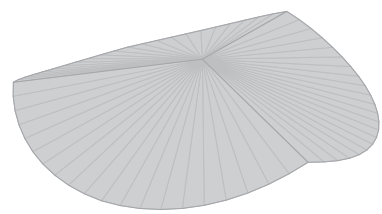

(a) $\Psi=-0.12 \beta$

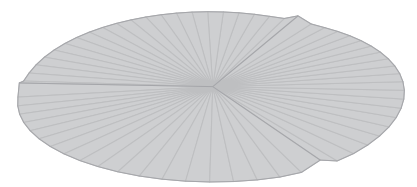

(e) $\Psi=0.10 \beta$

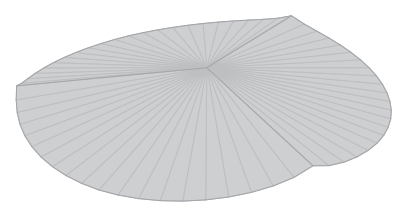

(b) $\Psi=-0.08 \beta$

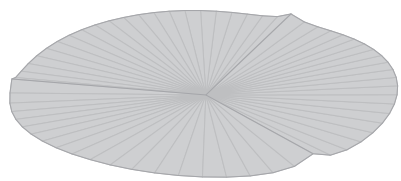

(f) $\Psi=0.20 \beta$

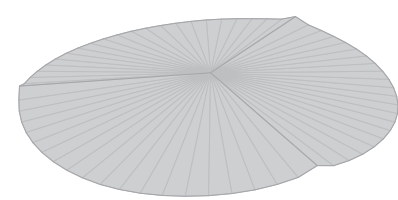

(c) $\Psi=-0.04 \beta$

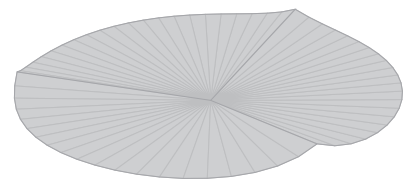

(g) $\Psi=0.30 \beta$

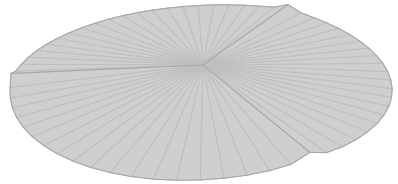

(d) $\Psi=0$

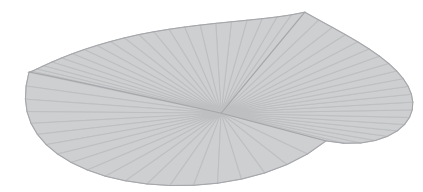

(h) $\Psi=0.40 \beta$

FIG. 9. Deformation sequence for a disk with three radial creases, $\beta=40^{\circ}, R=30, r=1, t=0.05$, and $n=10$. The deformed shapes shown in (b)- $(\mathrm{g})$ require separation of the facets, corresponding to membrane stretching of the faces. This separation is sufficiently small to not be visible. Comparing the stable states, (a) and (h), to Fig. 1 shows good qualitative agreement.

and stretch angle obtained from the previous step as an initial guess, the values for the rotation $\Psi+d \Psi$ are obtained using the same minimisation scheme. This process is continued to compute the complete strain energy-crease rotation behavior. Figure 9 shows the resulting deformation sequence for a disk with three radial creases $(N=3)$ and end rotations ranging between the two stable states at $\Psi=-0.12 \beta$ and $\Psi=0.40 \beta$. Comparing these to Fig. 1 shows good qualitative agreement.

The relationship between strain energy and end rotation for disks with $N=2$ to $N=5$ radial creases, for increasing numbers $(n)$ of hinge lines, are shown in Fig. 10. The diametrical crease disks $(N=2)$ are a special case since they have no strain energy at $\Psi=0$. In each case there are two energy minimums, corresponding to the stable states, separated by an energy peak. Predictions for the energy and end rotation at these stable states were obtained by Lechenault and AddaBedia [14] by finding minimums of bending energy while enforcing inextensibility. Their results are shown dashed in Fig. 10. As the number of hinges is increased, the present model rapidly approaches the stable state predictions made in Ref. [14]. The lowest stable state converges more quickly than the upper stable state with increasing $n$. Even for small numbers of hinges, the model converges quickly outside of the two stable states, since primarily bending deformations are involved and Eq. (6) provides a good approximation of the bending energy, even for moderate rotation angles.

The contributions of the bending and stretching energies to the total strain energy are shown in Fig. 11. Stretching has a large effect around $\Psi=0$ but diminishes quickly as the crease rotation is increased. The bending energy has two energy minimums and therefore drives the bistability of creased disks, while stretching increases the energy barrier between stable states. At the energy minimums the stretching energy is zero, since all the facets fit together without any gaps, as shown in Ref. [16].

To obtain the force required to move between equilibrium states, we note the total potential energy, $\Pi$, is

$$
\Pi=U_{T}-F \delta,
$$

where $U_{T}$ is the total strain energy [Eq. (21)], $F$ is an applied force acting along the global $z$ axis at the central vertex, and
$\delta=R \sin \Psi$ is the vertical displacement of the vertex. Since at equilibrium $d \Pi / d \delta=0$, we obtain an expression for the applied force:

$$
F=\frac{d U_{T}}{d \delta} .
$$

Using the strain energy-crease rotation behavior obtained previously, the force-displacement relationship is obtained using Eq. (23) and shown in Fig. 12 for disks with $N=$ 2 and $N=3$ radial creases. For the case of a diametrical crease $(N=2)$, shown in Fig. 12(a), no force is required to maintain the initial state $(\delta=0)$. An increase in displacement requires an increase of the applied force. The force reaches a peak before quickly dropping to a maximum negative value, which is much smaller in magnitude than the peak positive force. The first crossing with $F=0$ corresponds to the total energy peak in Fig. 10(a). Increasing the displacement further requires a slowly decreasing force magnitude until a second zero crossing is reached, which corresponds to the second stable state, or energy minimum in Fig. 10(a).

For $N>2$ the initial state $(\delta=0)$ requires a downward force to maintain. Starting from the lowest energy stable state, increasing the displacement requires an increase in the downward force. Like the $N=2$ disk, a peak is reached before quickly dropping to the maximum negative value. Continued displacement requires a gradually decreasing load magnitude until the second zero crossing is reached, corresponding to the second stable state. Further displacement requires a slowly increasing downward force.

This model assumes that the radial creases, and hinge lines, remain straight. However, as described in Sec. II, experiments show that a localized dimple forms around the indenter and grows in size as indentation continues. We now modify this rigid-faceted approach to incorporate this observed behavior.

\section{B. Bending crease}

If bending is allowed along the crease line, then an alternative deformation mode characterized by a localized dimple around the indentation point occurs, as described in Sec. II. Following the rigid-faceted approach, we discretize each face 


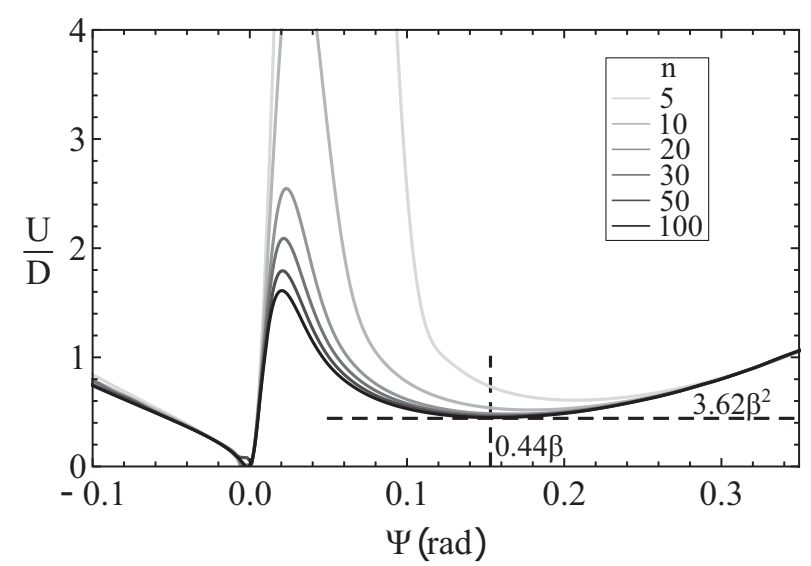

(a) $\mathrm{N}=2$

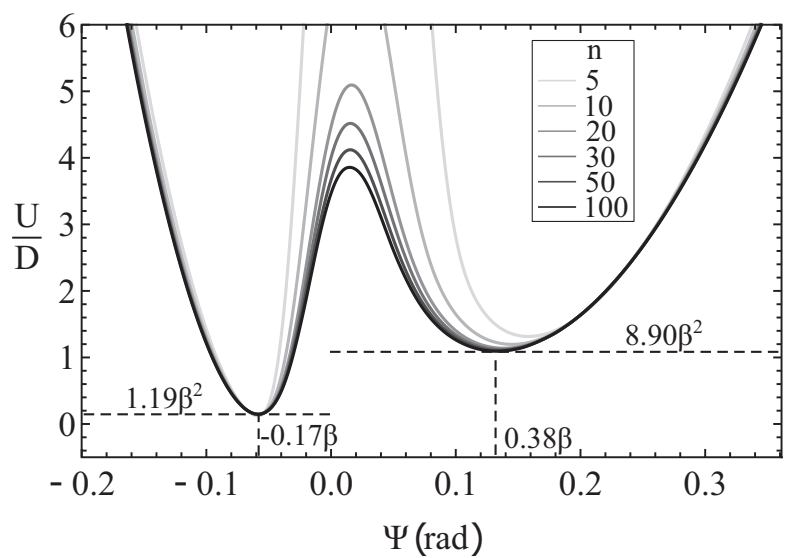

(c) $\mathrm{N}=4$

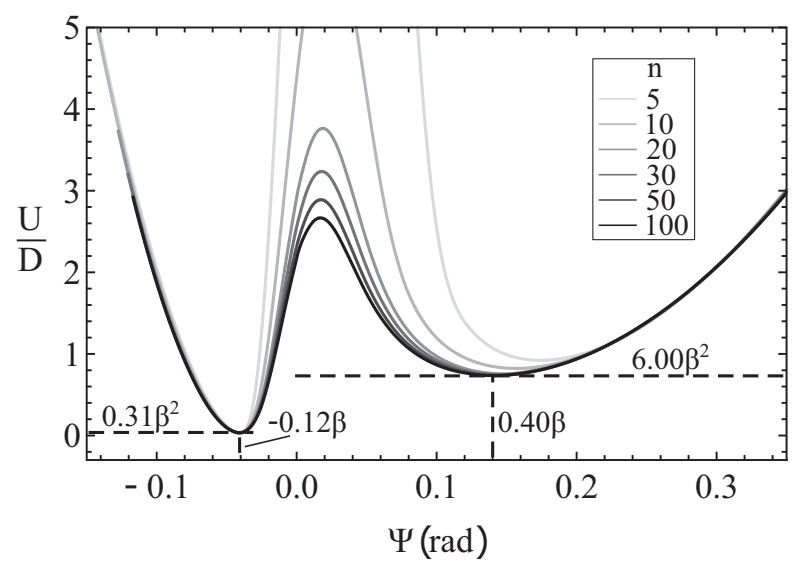

(b) $\mathrm{N}=3$

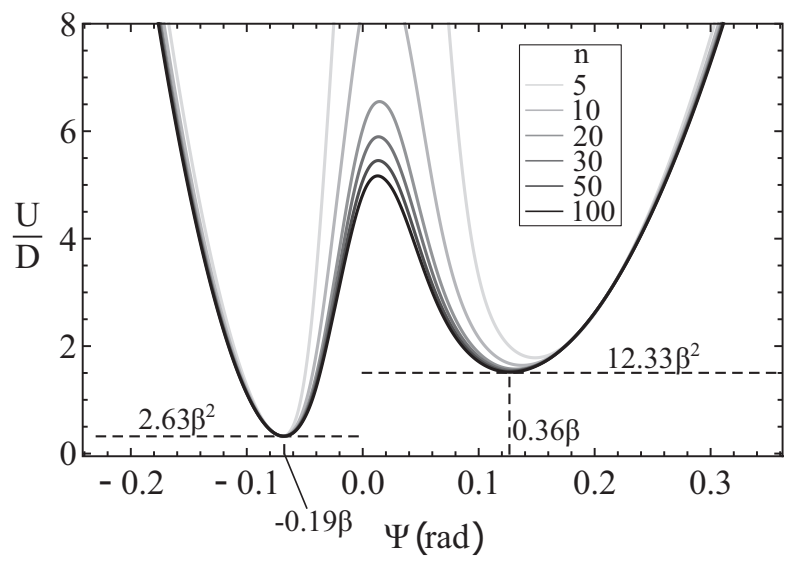

(d) $\mathrm{N}=5$

FIG. 10. Effect of the number of hinges on the strain energy-crease rotation behavior of creased disks with $\beta=20^{\circ}, R=30, r=1$, and $t=0.05$. As the number of hinges is increased the energy minimums rapidly approach the stable state predictions from Ref. [14], shown dashed.

between creases into a series of rigid facets and postulate a kinematic mechanism which captures the dimple deformation. The postulated facet layout is shown in Fig. 13. There are two key differences in comparison to the straight-crease model (Fig. 6). First, there is a circumferential hinge located at a radius of $\Delta$, measured along the crease from the center of

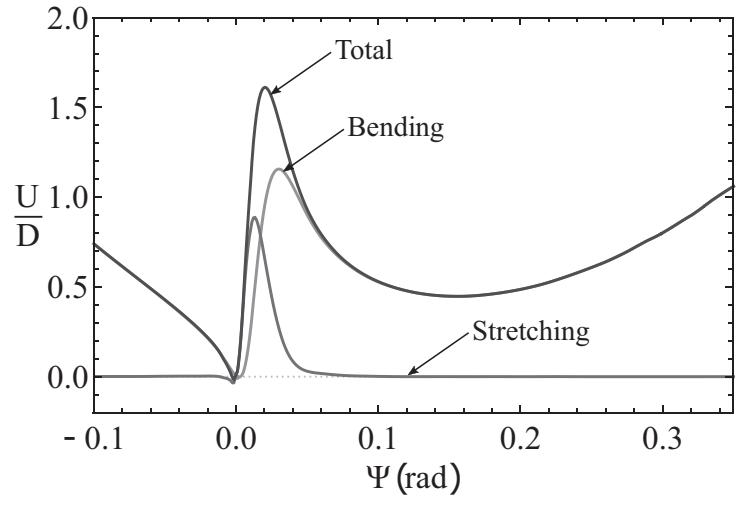

(a)

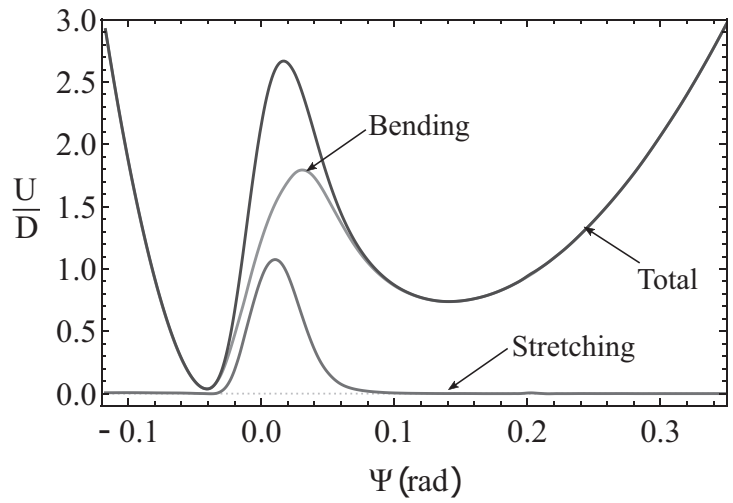

(b)

FIG. 11. Contributions of bending and stretching energy to the total strain energy of disks with $N=2$ (a) and $N=3$ (b) radial creases and $n=100, \beta=20^{\circ}, R=30, r=1$, and $t=0.05$. Stretching has a large effect around $\Psi=0$ but diminishes quickly outside this region. Bistability is governed by the bending energy, which has two minimums. 


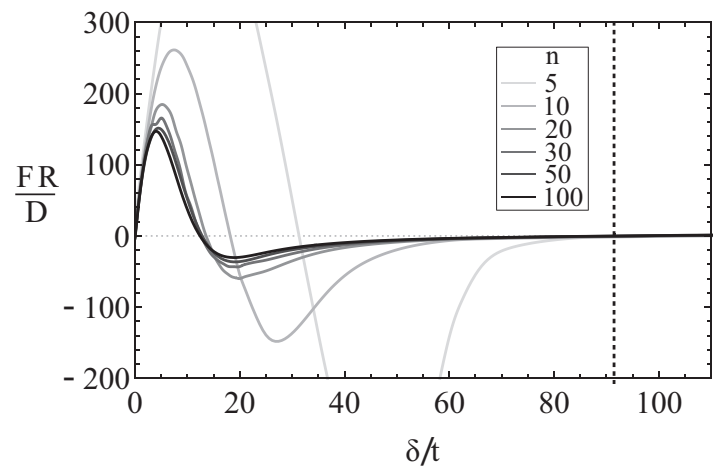

(a) $\mathrm{N}=2$

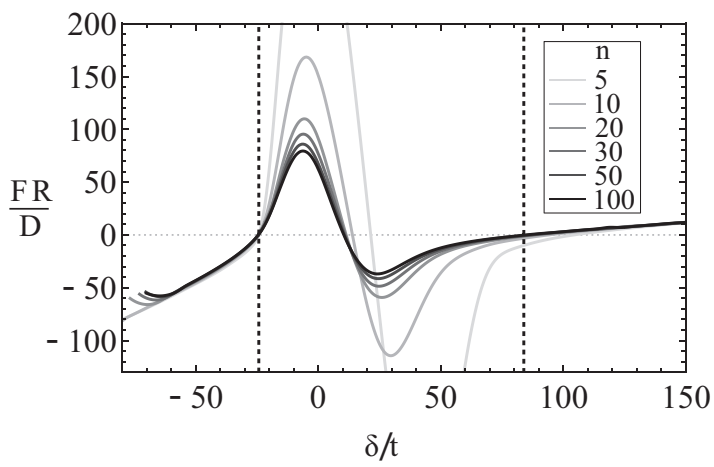

(b) $\mathrm{N}=3$

FIG. 12. Effect of the number of hinges on the force-displacement behavior of a creased disk with $N=2$ (a) and $N=3$ (b) radial creases and $\beta=20^{\circ}, R=30, r=1$, and $t=0.05$. The vertical dotted lines indicate the stable states corresponding to $F=0$.

the disk. Second, there are additional facets which emanate from the intersection of this hinge with the crease line (facets $\mathrm{C}$ and $\mathrm{D})$. We assume that the facets directly adjacent to the crease (A-F) have subtend angles of $\alpha_{0}$, while all other facets have subtended angles of $\alpha=\left(\pi-2 \alpha_{0}\right) /(2 n-1)$. Like the straight-crease model, we consider the kinematics before deriving the strain energy of deformation for this model.

\section{Kinematic analysis}

Deformation of this facet arrangement does not require stretching between facets. Therefore, we use a Gauss mapping approach to obtain the relationship between hinge rotations at each vertex. This technique maps the rotations of hinges intersecting at a single vertex onto a unit, or Gauss, sphere. As a result, the length of curves on the map are equal to the hinge rotations. If the signed enclosed area of the spherical polygon formed by these hinge rotations is equal to zero, then the vertex is developable-or more directly for our case, all facets intersecting at the vertex fit together without gaps [23]. This provides the condition for compatibility of the vertices.

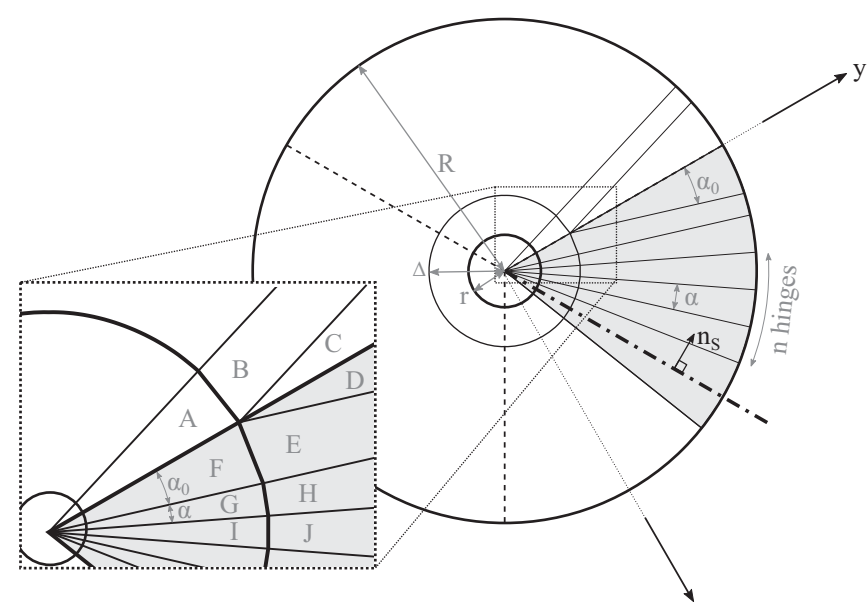

FIG. 13. Crease-bending model facet layout. A circumferential hinge is located at a distance $\Delta$ from the central vertex. This layout deforms by rotation of the hinge lines only.
We assume the crease angle in the outer region (between facets $C$ and $D$ ) remains fixed at $\beta$. The kinematics then requires the crease angle in the inner region (between facets $A$ and $F$ ) to change to accommodate any deformation. For the kinematic analysis we consider the vertices surrounded by facets $A B C D E F, E F G H$, and HGIJ. Since, as shown by Seffen [24], all further vertices around the ridge will result in the identical hinge rotation relationships as HGIJ. In particular, rotation $H J=-G I$ and $G H=I J$. This is a discrete result of developable curved folding [25].

Gauss maps for the vertices $A B C D E F, E F G H$, and $H G I J$ are shown in Fig. 14. Note that these are projections of spherical maps onto a tangent 2D surface; however, all calculations have been done for the spherical geometry. To ensure compatibility at the vertices, spherical areas $S_{1}+S_{3}=S_{2}, S_{4}=S_{5}$, and $S_{6}=S_{7}$. Full details of the kinematic calculations are included in Appendix A and summarized in Table I.

We impose a central displacement, $\delta$, which adds a constraint on the hinge rotations such that $\delta=\Delta \sin (\varangle A a C)$. This constraint is used to solve for the hinge rotation $D E$ (see full

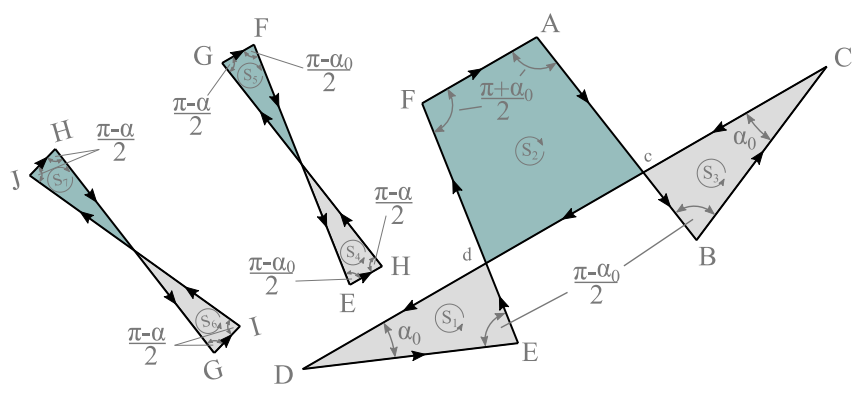

FIG. 14. Planar projection of the bending crease model Gauss maps. For compatibility of each vertex the signed enclosed area of each spherical polygon must equal zero. Therefore, $S_{1}+S_{3}=S_{2}$, $S_{4}=S_{5}$, and $S_{6}=S_{7}$. Since the Gauss map is on a sphere, the nominally parallel lines $A F$ and $C D$ intersect at two poles. We label one of these poles point $a$. We label the intersection of lines $E F$ and $A B$ as point $b$. 
TABLE I. Bending crease model kinematic relationships.

\begin{tabular}{|c|c|}
\hline Variable & Expression \\
\hline$\alpha_{0}, \delta, \Delta$ & Parameters \\
\hline$\alpha$ & $\frac{\pi-2 \alpha_{0}}{2 n-1}$ \\
\hline$\varangle D d E=\varangle C c B=\varangle A c D=\varangle F d C$ & $\sin ^{-1}\left[\cos \left(\frac{\alpha_{0}}{2}\right) \sqrt{\left\{1-[1-\cos (D E)] \cos \left(\alpha_{0}\right)\right\}^{2}+\sin ^{2}(D E)}\right]$ \\
\hline$C c=D d$ & $\cot ^{-1}\left[\frac{1}{\sin (D E)}-\cos \left(\alpha_{0}\right) \tan \left(\frac{D E}{2}\right)\right]$ \\
\hline$c d$ & $\beta-2 C c$ \\
\hline$\varangle A a C$ & 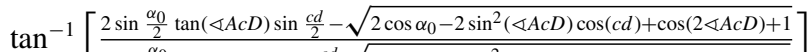 \\
\hline समान & {$\left[2 \sin \frac{\alpha_{0}}{2}+\tan (\varangle A c D) \sin \frac{c d}{2} \sqrt{2 \cos \alpha_{0}-2 \sin ^{2}(\varangle A c D) \cos (c d)+\cos (2 \varangle A c D)+1}\right]$} \\
\hline$A c=F d$ & $\cos ^{-1}\left[\frac{\cos (\varangle A a C)-\sin \left(\frac{\alpha_{0}}{2}\right) \cos (\varangle A c D)}{\cos \left(\frac{\alpha_{0}}{2}\right) \sin (\varangle A c D)}\right]$ \\
\hline$B c=E d$ & $\sin ^{-1}\left(2 \sin \left(\frac{\alpha_{0}}{2}\right)\left\{\left[\frac{1}{\sin (\mathrm{DE})}-\cos \left(\alpha_{0}\right) \tan \left(\frac{\mathrm{DE}}{2}\right)\right]^{2}+1\right\}^{-\frac{1}{2}}\right)$ \\
\hline$A B=E F$ & $B c+A c$ \\
\hline$\varangle c b d$ & $\cos ^{-1}\left[\sin ^{2}(\varangle A c D) \cos (c d)-\cos ^{2}(\varangle A c D)\right]$ \\
\hline$b d=b c$ & $\sin ^{-1}\left[\frac{\sin (\varangle A c D) \sin (c d)}{\sqrt{1-\left[\cos ^{2}(\varangle A c D)-\sin ^{2}(\varangle A c D) \cos (c d)\right]^{2}}}\right]$ \\
\hline$A F$ & $\cos ^{-1}\left[\cos ^{2}(b c-A c)+\sin ^{2}(b c-A c) \cos (\varangle c b d)\right]$ \\
\hline$H G$ & $2 \sin ^{-1}\left[\frac{\cos \left(\alpha_{0} / 2\right) \sin (E F / 2)}{\cos (\alpha / 2)}\right]$ \\
\hline$F G=E H$ & $2 \tan ^{-1}\left[\frac{\sin \left(\frac{\alpha+\alpha_{0}}{4}\right) \tan \left(\frac{E F+H G}{4}\right)}{\cos \left(\frac{\alpha-\alpha_{0}}{4}\right)}\right]$ \\
\hline$G I \ldots$ & $2 \tan ^{-1}\left[\sin \left(\frac{\alpha}{2}\right) \tan \left(\frac{H G}{2}\right)\right]$ \\
\hline$I J \ldots$ & $H G$ \\
\hline$H J \ldots$ & $G I$ \\
\hline
\end{tabular}

calculation in Appendix A):

$$
D E=\tan ^{-1}\left(\frac{c_{1} c_{2}+c_{3} \sqrt{c_{1}^{2}+c_{2}^{2}-c_{3}^{2}}}{c_{1}^{2}-c_{3}^{2}}\right),
$$

where

$$
\begin{aligned}
c_{1} & =\delta \cos \left(\frac{\alpha_{0}}{2}\right) \cos \left(\frac{\beta}{2}\right), \\
c_{2} & =\cos \frac{\alpha_{0}}{2}\left(\delta \cos \alpha_{0} \sin \frac{\beta}{2}+\sin \alpha_{0} \sqrt{\Delta^{2}-\delta^{2}}\right), \\
c_{3} & =\sin \frac{\alpha_{0}}{2}\left(\Delta-\delta \sin \alpha_{0} \sin \frac{\beta}{2}+\cos \alpha_{0} \sqrt{\Delta^{2}-\delta^{2}}\right) .
\end{aligned}
$$

Unlike the previous model, all hinge rotations are a function of only three parameters: $\alpha_{0}, \delta$, and $\Delta$. We solve for the deformed shape by fixing the values of $\alpha_{0}$ and $\delta$ then solving for $\Delta$ using the symmetry boundary condition. To obtain the mechanical behavior we also require the corresponding strain energy of deformation.

\section{Energy}

The facet bending energy for a wedge-shaped facet is given by Eq. (10). However, facet $E$ is rectangular. It is straightforward to show that its bending energy is

$$
\left(U_{B}\right)_{E}=\frac{2(R-\Delta)}{\Delta \alpha_{0}} D E^{2} .
$$

The deformation of facet $F$ is due to the change in the angle of the inner crease $(\beta-A F)$. The facets within the ridge have an outer radius equal to the ridge radius, $\Delta$, and an inner radius equal to the hole radius, $r$. The bending energy of facet $F$ due to the opening of the inner crease is obtained using Eq. (10):

$$
\left(U_{B}\right)_{F}=D \frac{\left(1+\cos \alpha_{0}\right)}{2\left(\alpha_{0}+\sin \alpha_{0}\right)} \ln \left(\frac{\Delta}{r}\right)\left(\frac{\beta-A F}{2}\right)^{2} .
$$

Facet $G$ is deformed by the hinge rotation $F G$, while all remaining facets within the ridge are deformed by a hinge rotation $G I$ :

$\left(U_{B}\right)_{i}=D \frac{(1+\cos \alpha)}{2(\alpha+\sin \alpha)}\left[\ln \left(\frac{\Delta}{r}\right)\left(F G^{2}+(n-1) G I^{2}\right)\right]$.

Outside the ridge the facet $E$ is deformed by the hinge rotation $E H$ and the remaining outer facets are deformed by the hinge rotation $H J$ :

$\left(U_{B}\right)_{o}=D \frac{(1+\cos \alpha)}{2(\alpha+\sin \alpha)}\left[\ln \left(\frac{R}{\Delta}\right)\left(E H^{2}+(n-1) H J^{2}\right)\right]$.

The total facet bending energy in one half face is the sum of these energies:

$$
\left(U_{B}\right)_{f}=\left(U_{B}\right)_{E}+\left(U_{B}\right)_{F}+\left(U_{B}\right)_{i}+\left(U_{B}\right)_{o} .
$$

The final energy component is the energy cost of the ridge. Inextensibility of this deformation mode requires vanishing disk thickness. For finite thickness there is a balancing of stretching and bending energies in the region of the ridge. Previous studies have shown that this deformation energy scales with $(L / t)^{1 / 3}$, where $L$ is the length of the ridge and $t$ is the thickness [26-28]. Further investigation of the forming and propagation of a localized ridge is beyond the scope of 


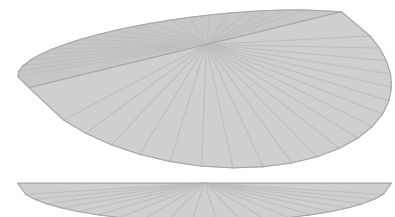

(a) $\delta=0 \mathrm{~mm}$

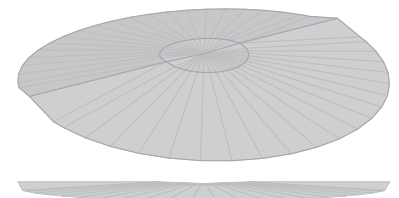

(b) $\delta=0.5 \mathrm{~mm}$

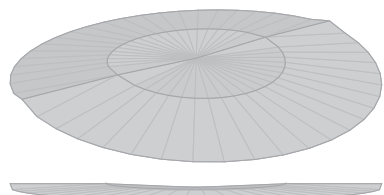

(c) $\delta=1.0 \mathrm{~mm}$

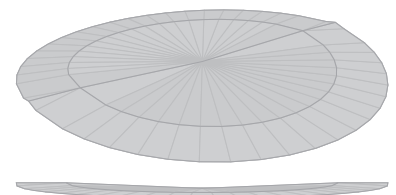

(d) $\delta=1.5 \mathrm{~mm}$

FIG. 15. Bending crease mode deformation sequence for a diametrically creased disk $(N=2)$ with $\beta=24^{\circ}, R=30, r=0.5, t=0.1$, $\alpha_{0}=17^{\circ}$, and $n=10$. As the indentation is increased the ridge expands radially towards the edge of the disk.

this work. Instead we propose the ridge energy as [28]

$$
\left(U_{B}\right)_{r}=C_{r}\left(\frac{\Delta}{t}\right)^{1 / 3}\left[\frac{\pi}{2}-\gamma\right]^{7 / 3},
$$

where $\gamma$ is the bending angle across the ridge. The constant of proportionality, $C_{r}$, is left as a fitting parameter. This does not consider the boundary effect when the ridge is near the central hole or the outer edge of the disk. We do not explicitly address this here but note it will have an effect on the predicted behavior.

The total strain energy is the sum of the facet bending energy and the energy of the ridge:

$$
U=2 N\left[\left(U_{B}\right)_{f}+\left(U_{B}\right)_{r}\right] .
$$

\section{Mechanical behavior}

The bending crease model has three geometric parameters, $\alpha_{0}, \delta$, and $\Delta$, plus the constant of proportionality $C_{r}$. We assume values for $\alpha_{0}$ and $C_{r}$, which remain constant for a particular disk, and solve for the ridge radius, $\Delta$. To obtain the mechanical behavior we begin with all creases lying in the $x$ - $y$ plane $(\delta=0)$. A vertex displacement, $\delta$, is imposed and the ridge radius, $\Delta$, obtained numerically by enforcing the symmetry boundary condition [Eq. (5)] using MATLAB [22]. The corresponding strain energy is computed using Eq. (31). The indentation depth is then incremented by a small amount, $d \delta$. Using the ridge radius obtained from the previous step as an initial guess, the radius corresponding to an indentation of $\delta+d \delta$ is obtained using the same numerical scheme. This process is continued to compute the complete strain energy-indentation behavior. Figure 15 shows the resulting deformation sequence for a disk with a diametrical crease $(N=2)$. Under increasing indentation, the ridge radius increases linearly (see Fig. 16) until it reaches the outer edge, when the disk snaps to the inverted state.

Using the strain energy-indentation results, the indentation force is calculated using Eq. (23). The change of energy and indentation force, as a function of indentation depth, are shown in Fig. 17. Under increasing indentation, the energy increases monotonically until the ridge reaches the outer edge of the disk. Figure 17(a) shows how the response varies with changes in the proportionality constant $C_{r}$. As it increases, both the energy cost and the indentation force increase. Conversely, as shown in Fig. 17(b), as $\alpha_{0}$ is increased both the indentation force and energy decrease. Additionally, as $\alpha_{0}$ increases, the indentation when the ridge reaches the disk edge also increases. Since there is no stretching energy, increasing the number of hinges has a negligible effect on the results.

\section{COMPARISON OF ANALYSIS AND EXPERIMENTS}

To compare the predictions from the models proposed in this study to experiments, we must adjust the prediction to match the loading and support conditions of the tests. In particular, the span of the supports and the size of the indenter. This requires a transformation from the model displacement coordinate, $\delta$, to the measured displacement coordinate $\delta^{*}$. It can be shown (see Appendix B) for the diametrically $(N=2)$ creased disk that

$$
\delta_{1}^{*}=\frac{\delta\left(L-d_{i}\right)}{2 R}, \quad \delta_{2}^{*}=\delta\left(1-\frac{d_{i}}{2 \Delta}\right),
$$

where $\delta_{1}^{*}$ and $\delta_{2}^{*}$ are the predicted test displacements of the straight crease and bending crease models, respectively, $L=$ $80 \mathrm{~mm}$ is the support span, $d_{i}=4 \mathrm{~mm}$ is the diameter of the indenter, $R=50 \mathrm{~mm}$ is the radius of the disk, and $\Delta$ is the dimple radius, measured along the crease.

For disks with three radial creases, the measured displacement is relative to the lower stable state. The contact point also changes from the hole edge to the indenter edge when the disk passes through the horizontal plane $(\delta=0)$. Since the angle between the creases and the horizontal plane in the lowest energy stable state is $0.12 \beta$ [14], the transformed displacement for the $N=3$ case is (see Appendix B):

$$
\begin{aligned}
\delta_{3}^{*}= & \frac{L}{2} \tan (0.12 \beta)-r \sin (0.12 \beta) \\
& +\frac{\delta}{2 R} \begin{cases}(L-2 r) & \text { if } \delta<0, \\
\left(L-d_{i}\right) & \text { if } \delta>0\end{cases}
\end{aligned}
$$

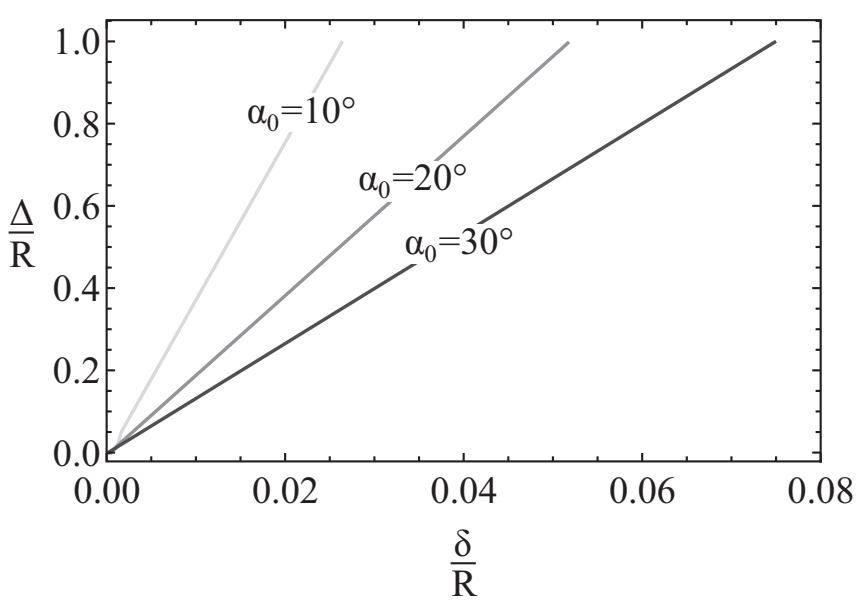

FIG. 16. Relationship between the indentation, $\delta$, and the dimple radius measured along the crease, $\Delta$. This relationship is driven entirely by the kinematics of the system and the symmetry boundary condition [Eq. (5)]. 


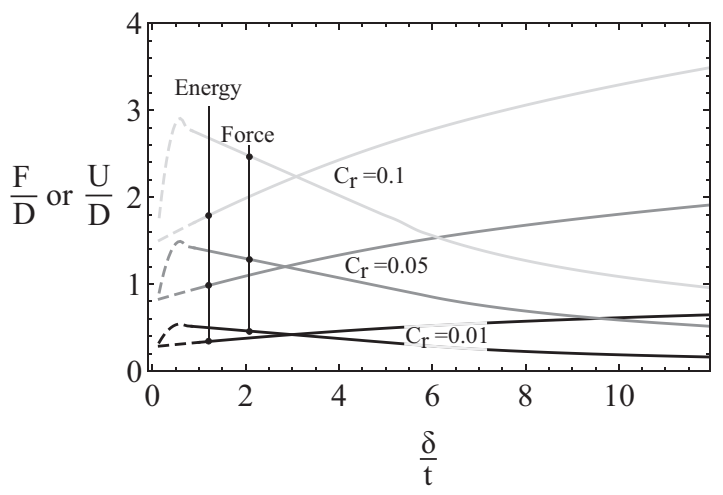

(a)

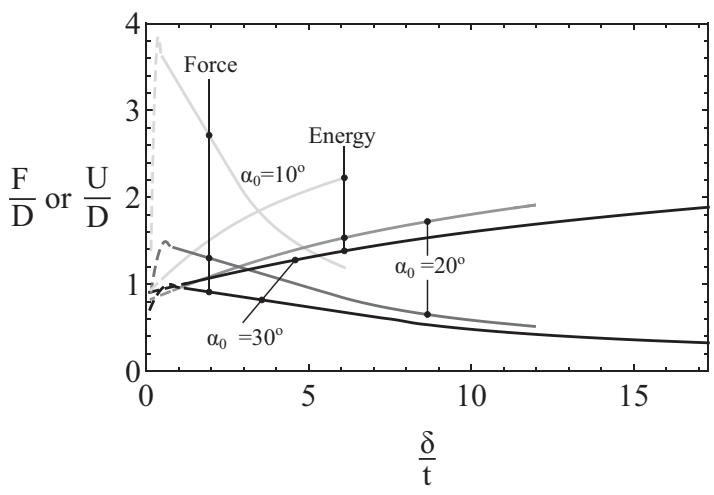

(b)

FIG. 17. Effect of parameters (a) $C_{r}$ and (b) $\alpha_{0}$ on predicted behavior for a disk with $N=2, R=30, \beta=20^{\circ}, r=1$, and $n=100$. Predictions when the ridge is located within two hole radii of the center have been shown dashed since boundary effects around the hole have not been considered.

These transformed displacements must also be used in Eq. (23) to obtain the indentation force.

Predictions from the two models are plotted with the experimental results and shown in Fig. 18. The ridge energy constant of proportionality, $C_{r}$, and hinge angle, $\alpha_{0}$, were used as fitting parameters for the bending crease model. To match the predictions of when the dimple reaches the disk edge with the experimentally observed snap-through, $\alpha_{0}$ was found to be approximately $9 \pi /(50 N)$. The constant of proportionality, $C_{r}$ was found to increase with the crease angle $\beta$.

The straight crease model predicts a much higher peak load than measured experimentally. This is due to the forming and propagation of the dimple, which is captured by the crease bending model. Since the forming and propagation of the dimple is bending dominated, it has a lower energy cost in comparison to the initially stretching dominated straightcrease deformation mode. Once the dimple has formed, the deformation cannot snap to the straight-crease form until the dimple reaches the disk edge. Therefore, the deformation ultimately ends up on a higher load path before suddenly snapping to the straight-crease form when the dimple reaches the disk edge. After snap-through the response closely matches the straight crease model.

\section{CONCLUSION}

In this study we considered the mechanics of a thin disk decorated by multiple radial creases both experimentally and analytically. We showed that the disks deform by forming a dimple under the point of indentation. Under further indentation this dimple grows in size until it reaches the disk edge, when the disk snaps to the inverted shape. Previous studies of this f-cone assumed straight creases, and generator lines, emanating from a central vertex which cannot capture this observed behavior.

Two models were derived to capture the observed deformation. We discretized the region between creases into a series of rigid facets connected by hinges that can both rotate and stretch. After deriving the kinematics for an arbitrary number of facets, the strain energy of deformation was calculated by imposing the hinge deformations as boundary conditions on continuum shell deformations of the facets. The complete mechanical behavior was obtained by minimizing the total strain energy subject to the hinge deformations.

The first model assumed the creases, and hinges, remain straight. We allow the facets to separate to capture membrane stretching. As the number of facets is increased, the results approach the predictions for the stable states made in previous studies. We therefore capture the entire mechanical behavior corresponding to the straight-crease assumption. However, when compared to experiments, this straight-crease model predicts much higher peak loads and earlier snap-through than observed experimentally. This is due to the forming and propagation of a localized dimple around the indentation point.

A second model was derived to allow for this dimple. Starting from the straight-crease model, we add a circumferential hinge at an arbitrary distance from the center. We derived the underlying kinematic mechanism using a Gauss mapping approach. In the limit of vanishing thickness the deformation is inextensible and thus only requires rotation of the hinges. The mechanical behavior predicted by the bending-crease model closely matches experiments up until snap-through while the straight-crease model captures the post-snap-through behavior. A combination of the two rigidfaceted models capture the complete mechanical behavior of creased disks.

The analysis approach derived in this study can be applied to other large-deformation shell mechanics problems by adopting an appropriate kinematic mechanism. It thereby connects the kinematic analysis of rigid origami to the continuum mechanics of thin shells, offering an effective new method for understanding the behavior of folded structures.

\section{ACKNOWLEDGMENTS}

M.G.W. is generously supported by the Mary Ewart Junior Research Fellowship at Somerville College, University of Oxford. This research was funded by the John Fell Oxford University Press Research Fund. The author also thanks M. A. Dias for fruitful discussions. 

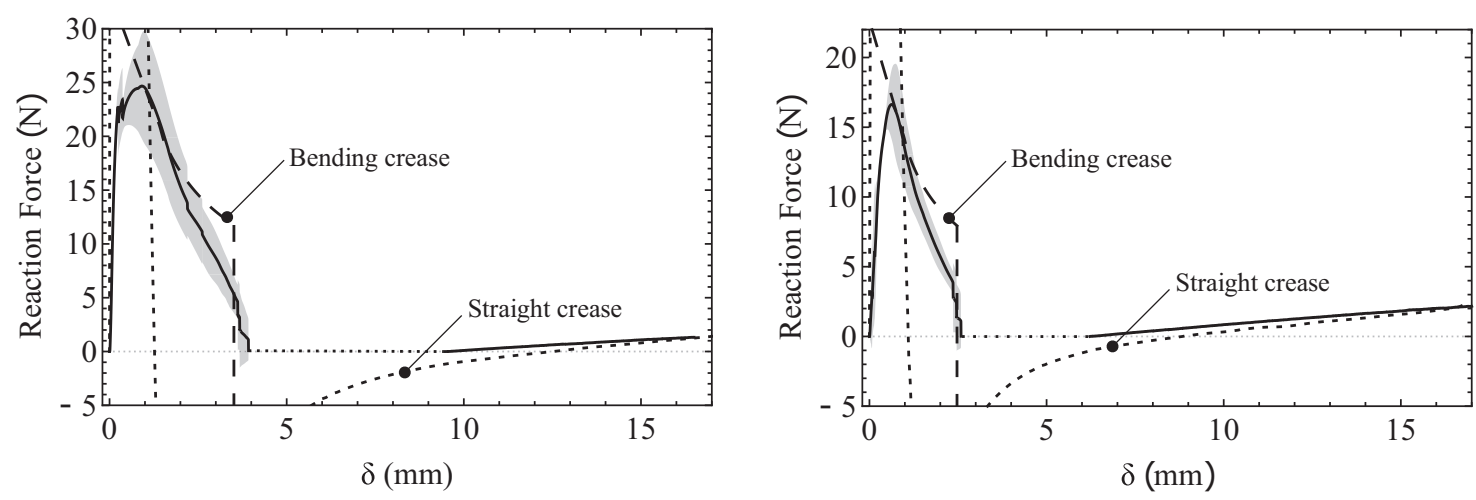

(a) $\mathrm{N}=2, \beta=44^{\circ}, \mathrm{C}_{\mathrm{r}}=2.1, \alpha_{0}=18^{\circ}$

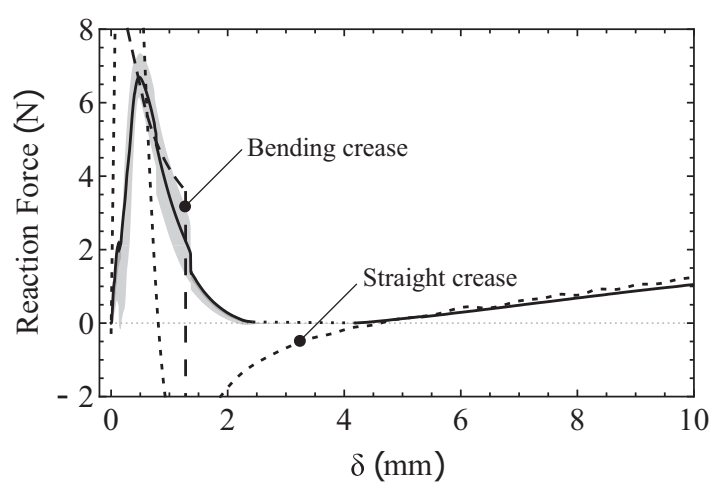

(b) $\mathrm{N}=2, \beta=31^{\circ}, \mathrm{C}_{\mathrm{r}}=0.93, \alpha_{0}=17^{\circ}$

(c) $\mathrm{N}=2, \beta=17^{\circ}, \mathrm{C}_{\mathrm{r}}=0.20, \alpha_{0}=17^{\circ}$

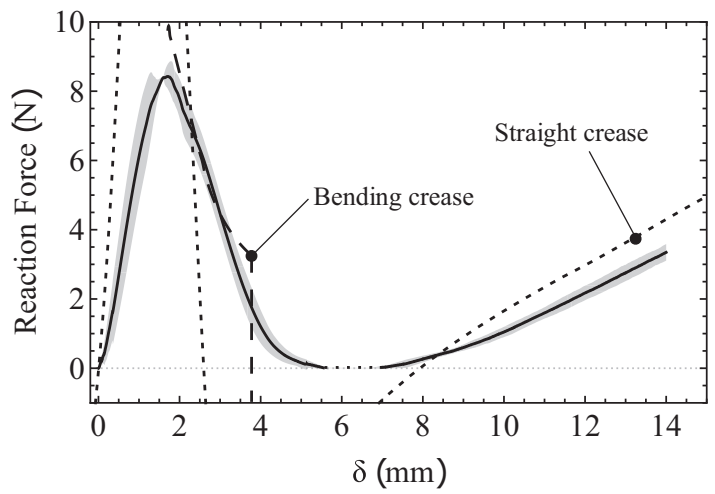

(d) $\mathrm{N}=3, \beta=23^{\circ}, \mathrm{C}_{\mathrm{r}}=0.28, \alpha_{0}=12^{\circ}$
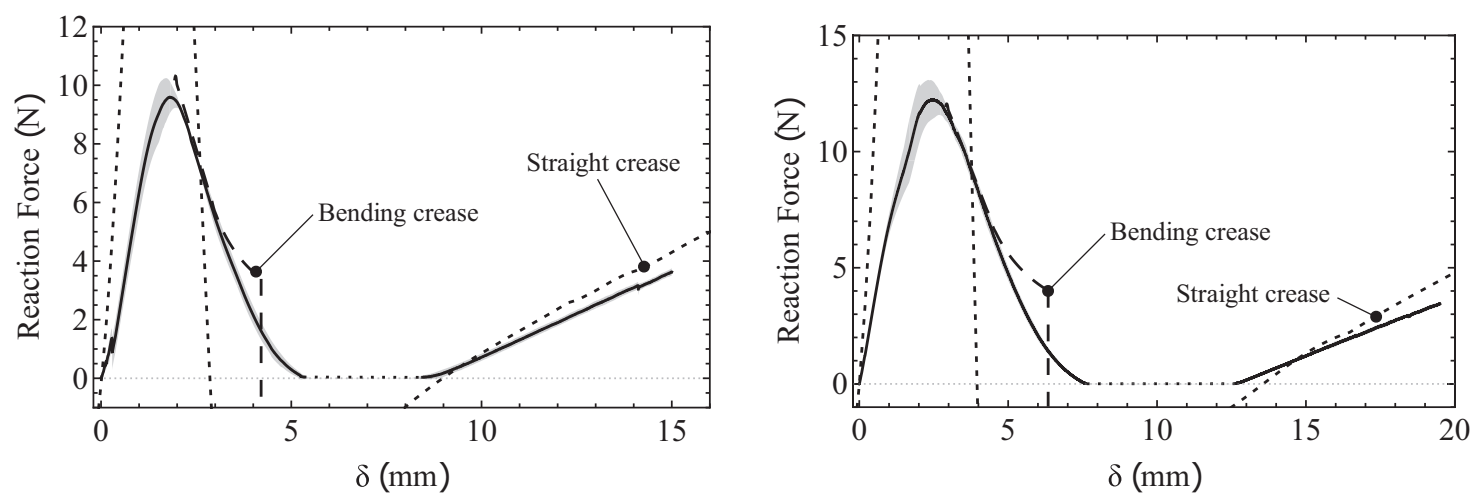

(e) $\mathrm{N}=3, \beta=26^{\circ}, \mathrm{C}_{\mathrm{r}}=0.32, \alpha_{0}=11^{\circ}$

(f) $\mathrm{N}=3, \beta=39^{\circ}, \mathrm{C}_{\mathrm{r}}=0.60, \alpha_{0}=11^{\circ}$

FIG. 18. Comparison of experiments and model predictions for disks with $N=2$ (a-c) and $N=3$ (d-f) creases. Each disk has $R=50 \mathrm{~mm}$, $r=1.5 \mathrm{~mm}, t=0.1 \mathrm{~mm}$ and is made from shim steel with $E=185 \mathrm{MPa}$ and $v=0.3$. Initial indentation behavior is captured by the bending crease model while the post snap-through behavior matches the straight-crease prediction well.

\section{APPENDIX A: CREASE BENDING MODEL KINEMATIC ANALYSIS}

Since this model deforms through rotations of the hinges only, facet separation is not permitted. Therefore, all facets intersecting at each vertex must fit together without gaps. This compatibility condition is enforced by ensuring the solid angle, and thereby the Gaussian curvature, at each vertex is zero using a Gauss mapping approach.

We translate the normal vector of each facet meeting at a particular vertex to the center of a unit sphere and mark the intersection of this vector with the surface. Moving from one facet to the next involves rotating the normal about the connecting hinge line, tracing out an arc of a great circle on the surface of the unit sphere. Since the sphere has a unit radius, the length of the arcs is equal to the relative rotation of the normal vectors across hinge lines [23,29]. Each arc has a direction given by the sense of rotation about the hinges. The arcs form spherical polygons and the enclosed regions are given a sign according to the circulation direction of the boundary. For example, polygons with a clockwise circulation direction can be considered positive and anti-clockwise 


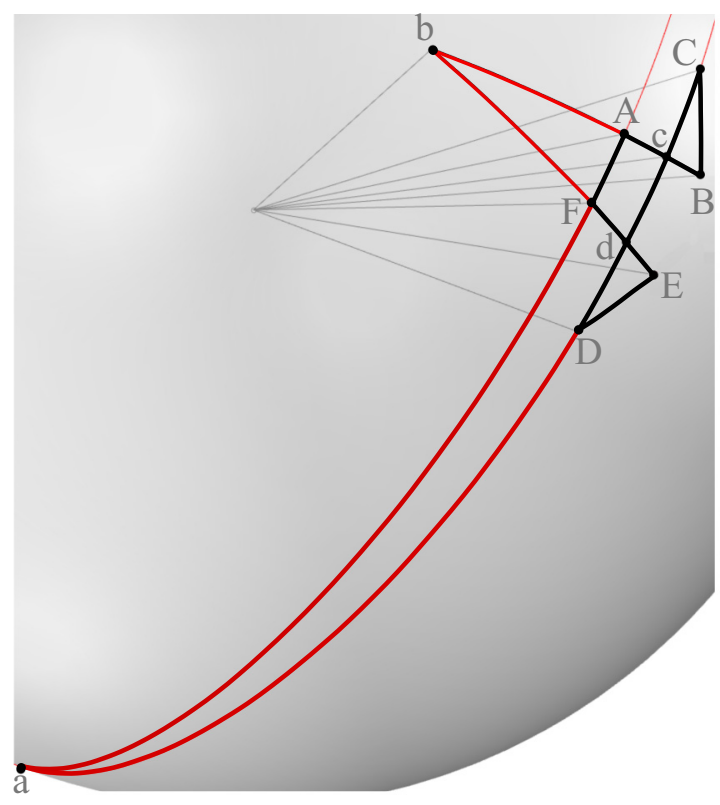

FIG. 19. Bending crease model Gauss map. The planar projection of this Gauss map is shown in Fig. 14.

negative. The signed enclosed area measures the solid angle at the vertex which must equal zero for compatibility. With this constraint, we derive the kinematic relationship between the facet rotations. The following derivation references Figs. 14 and 19.

We require the relationship between hinge rotations, or the side lengths of the spherical polygons, which ensure compatibility at each vertex. We take $D E$ as a deformation parameter defined by the imposed center displacement and consider the compatibility of vertex $A B C D E F$ first.

\section{a. Vertex $A B C D E F$}

We assume that the outer region crease (between facets $\mathrm{C}$ and $\mathrm{D}$ ) does not open. Therefore the length $C D=\beta$. For compatibility the areas (see Fig. 14) $S_{1}+S_{3}=S_{2}$. We first find the areas $S_{1}=S_{3}$. Computing the length $D d=C c$ using the cotangent four-part formula:

$$
\begin{gathered}
\cos (D E) \cos \alpha_{0}=\cot (D d) \sin (D E)-\sin \alpha_{0} \cot \left(\frac{\pi-\alpha_{0}}{2}\right) \\
\longrightarrow D d=C c=\cot ^{-1}\left[\frac{1}{\sin (D E)}-\cos \left(\alpha_{0}\right) \tan \left(\frac{D E}{2}\right)\right] .
\end{gathered}
$$

Then using the spherical law of Sines:

$$
\begin{aligned}
& \sin (\varangle D d E) \\
& =\sin (\varangle C c B)=\frac{\sin D E \cos \left(\frac{\alpha_{0}}{2}\right)}{\sin D d} \\
& =\cos \left(\frac{\alpha_{0}}{2}\right) \sqrt{\left[1-(1-\cos (D E)) \cos \left(\alpha_{0}\right)\right]^{2}+\sin ^{2}(D E)} .
\end{aligned}
$$

The enclosed area, which is equal to the spherical excess, is

$$
S_{1}=S_{3}=\varangle D d E-\frac{\pi-\alpha_{0}}{2} .
$$

For compatibility $S_{1}+S_{3}=S_{2}$. Therefore, the enclosed area $S_{2}$ must be

$$
\begin{aligned}
S_{2}=2 \frac{\pi+\alpha_{0}}{2}+2 \varangle F d C-2 \pi & =2 S_{1} \\
\longrightarrow \varangle F d C & =\varangle D d E .
\end{aligned}
$$

Due to symmetry: $\varangle A c D=\varangle F d C=\varangle D d E$.

We define a point $a$ where the great circles containing $A F$ and $C D$ intersect, as shown in Fig. 19. Considering the spherical triangle $A a c$, and noting that the length of $a c$ is $(\pi+c d) / 2$, we use the spherical Cosine law to find $\varangle A a C$ :

$$
\begin{aligned}
\sin \left(\frac{\alpha_{0}}{2}\right) & =\sin (\varangle A a C) \sin (\varangle A c D) \sin \left(\frac{c d}{2}\right) \\
& +\cos (\varangle A a C) \cos (\varangle A c D) .
\end{aligned}
$$

Solving for $\varangle A a C$,

$$
\tan (\varangle A a C)=\frac{2 \sin \frac{\alpha_{0}}{2} \tan (\varangle A c D) \sin \frac{c d}{2}-\sqrt{2 \cos \alpha_{0}-2 \sin ^{2}(\varangle A c D) \cos (c d)+\cos (2 \varangle A c D)+1}}{2 \sin \frac{\alpha_{0}}{2}+\tan (\varangle A c D) \sin \frac{c d}{2} \sqrt{2 \cos \alpha_{0}-2 \sin ^{2}(\varangle A c D) \cos (c d)+\cos (2 \varangle A c D)+1}} .
$$

This enables us to solve for $A c=F d$ using the spherical cosine law again:

$$
\begin{aligned}
& \cos (\varangle A a C)=\sin \left(\frac{\pi+\alpha_{0}}{2}\right) \sin (\varangle A c D) \cos A c-\cos \left(\frac{\pi+\alpha_{0}}{2}\right) \cos (\varangle A c D) \\
& \longrightarrow \cos A c=\cos F d=\frac{\cos (\varangle A a C)-\sin \left(\frac{\alpha_{0}}{2}\right) \cos (\varangle A c D)}{\cos \left(\frac{\alpha_{0}}{2}\right) \sin (\varangle A c D)} .
\end{aligned}
$$

Moving to triangle $D d E$, the length $B c=E d$ is found using the spherical Sine law:

$$
\begin{gathered}
\frac{\sin E d}{\sin \alpha_{0}}=\frac{\sin D E}{\sin (\varangle D d E)} \\
\longrightarrow \sin (E d)=\sin (B c)=\sin ^{-1}\left(\frac{2 \sin \left(\frac{\alpha_{0}}{2}\right)}{\sqrt{\left[\frac{1}{\sin (\mathrm{DE})}-\cos \left(\alpha_{0}\right) \tan \left(\frac{\mathrm{DE}}{2}\right)\right]^{2}+1}}\right) .
\end{gathered}
$$




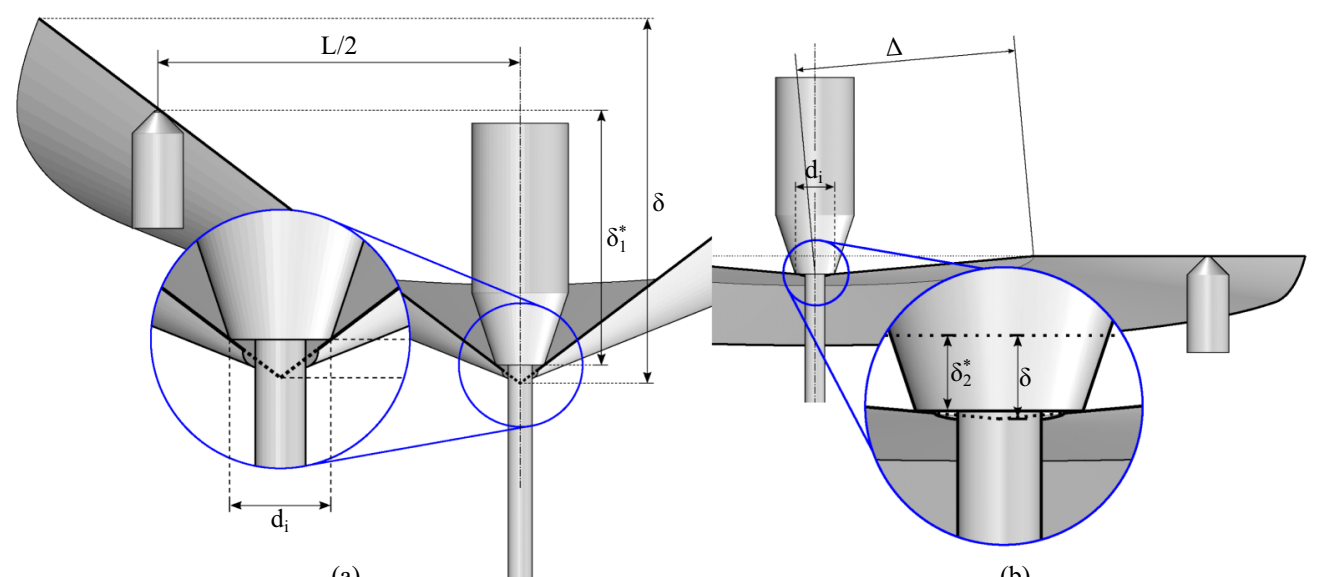

(a)

(b)

FIG. 20. Deformed cross-sections for the (a) straight crease and (b) bending crease deformation modes.

By combining Eqs. (A7) and (A8), we calculate

$$
A B=E F=B c+A c .
$$

We locate a point $b$ at the intersection of the great circles containing the arcs $A B$ and $E F$, as shown in Fig. 19. Then, considering triangle $c b d$ we find the length $b c$ by first using the spherical cosine law,

$$
\cos (\varangle c b d)=\sin ^{2}(\varangle A c D) \cos (c d)-\cos ^{2}(\varangle A c D),
$$

then the spherical sine law,

$$
\begin{aligned}
\frac{\sin (b c)}{\sin (\varangle A c D)} & =\frac{\sin (c d)}{\sin (\varangle c b d)} \\
\longrightarrow \sin (b c) & =\frac{\sin (\varangle A c D) \sin (c d)}{\sqrt{1-\left[\cos ^{2}(\varangle A c D)-\sin ^{2}(\varangle A c D) \cos (c d)\right]^{2}}} .
\end{aligned}
$$

Since $A b=F b=b c-A c$, we obtain the angle across the crease in the inner region, $A F$, using the spherical cosine law again,

$$
\cos (A F)=\cos ^{2}(A b)+\sin ^{2}(A b) \cos (\varangle c b d) .
$$

Next we consider the vertex $E F G H$.

\section{b. Vertex EFGH}

For compatibility of vertex $E F G H$ the areas $S_{4}=S_{5}$, from which we conclude by inspection that $F G=E H$. The length $E F$ was obtained previously [Eq. (A9)] by considering the vertex $A B C D E F$. We continue to solve for the remaining hinge rotations $H G$, and $F G=E H$. Using the spherical sine law,

$$
\begin{aligned}
\frac{\sin \left(\frac{E F}{2}\right)}{\sin \left(\frac{\pi-\alpha}{2}\right)} & =\frac{\sin \left(\frac{H G}{2}\right)}{\sin \left(\frac{\pi-\alpha_{0}}{2}\right)} \\
\longrightarrow H G & =2 \sin ^{-1}\left[\frac{\cos \left(\frac{\alpha_{0}}{2}\right) \sin \left(\frac{E F}{2}\right)}{\cos \left(\frac{\alpha}{2}\right)}\right] .
\end{aligned}
$$

Then, using Napier's analogy:

$$
\begin{aligned}
\tan \left(\frac{E F+H G}{4}\right) & =\frac{\cos \left(\frac{\alpha-\alpha_{0}}{4}\right)}{\cos \left(\frac{2 \pi-\alpha-\alpha_{0}}{4}\right)} \tan \left(\frac{F G}{2}\right) \\
\longrightarrow F G=E H & =2 \tan ^{-1}\left[\frac{\sin \left(\frac{\alpha+\alpha_{0}}{4}\right) \tan \left(\frac{E F+H G}{4}\right)}{\cos \left(\frac{\alpha-\alpha_{0}}{4}\right)}\right]
\end{aligned}
$$

Finally, we consider vertex $I J G H$.

\section{c. Vertex IJGH}

All further vertices have the identical geometry as vertex $I J G H$ since all internal angles of the Gauss map are the same. For compatibility $S_{6}=S_{7}$, therefore $J H=G I$ and $H G=J I$, which is a result from Ref. [24]. The length $H G$ was obtained from vertex $E F G H$; see Eq. (A13). We solve for $G I=J H$ using Napier's analogy:

$$
\begin{aligned}
\tan \frac{H G}{2} & =\frac{1}{\cos \frac{\pi-\alpha}{2}} \tan \frac{G I}{2} \\
\longrightarrow G I & =2 \tan ^{-1}\left[\tan \frac{H G}{2} \sin \frac{\alpha}{2}\right] .
\end{aligned}
$$

The imposed central displacement, $\delta$, provides an additional kinematic constraint which is used to obtain $D E$.

\section{d. Central displacement}

We use the central displacement, $\delta$, to obtain the hinge rotation $D E$, which the remaining angles depend on. The displacement is due to inclination of the central crease line (between facets $A$ and $F$ ) from the $x-y$ plane, since the outer crease (between facets $C$ and $D$ ) remains in the $x-y$ plane. The angle that the inner crease makes with the $x-y$ plane is equal to the angle between the outer crease line, $C D$, and the inner crease line, $A F$, on the Gauss map, or $\varangle A a C$. Therefore, we 


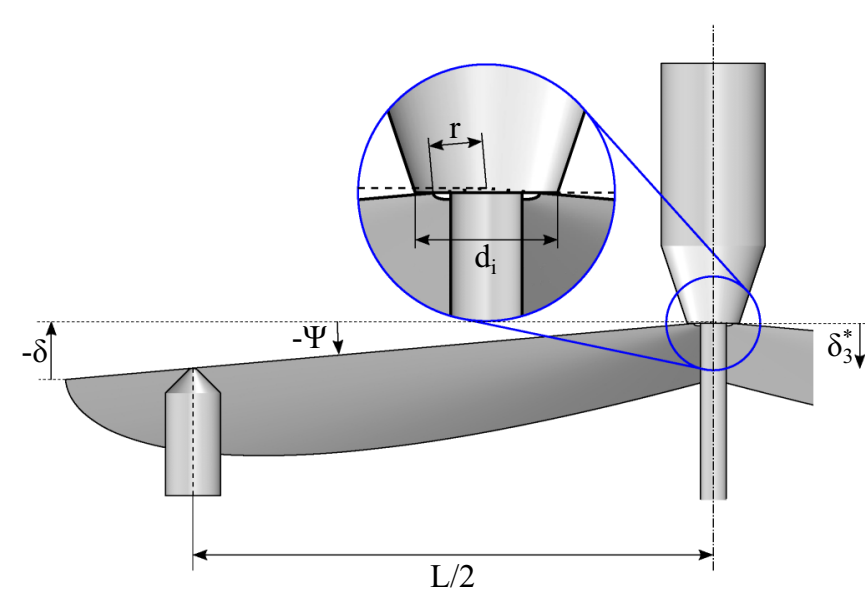

FIG. 21. Deformed cross-sections for the case of $N>2$, showing the natural shape.

can write

$$
\delta=\Delta \sin (\varangle A a C), \quad \sqrt{\Delta^{2}-\delta^{2}}=\cos (\varangle A a C) .
$$

Then, from Eq. (A5), noting that $c d=\beta-2 D d$,

$$
\begin{aligned}
& \delta \sin (\varangle A c D) \sin \left(\frac{\beta}{2}-D d\right) \\
& +\sqrt{\Delta^{2}-\delta^{2}} \cos (\varangle A c D)=\Delta \sin \left(\frac{\alpha_{0}}{2}\right) .
\end{aligned}
$$

After substituting Eqs. (A1) and (A2) into Eq. (A17) and solving for $D E$,

$$
D E=\tan ^{-1}\left(\frac{c_{1} c_{2}+c_{3} \sqrt{c_{1}^{2}+c_{2}^{2}-c_{3}^{2}}}{c_{1}^{2}-c_{3}^{2}}\right)
$$

where

$$
\begin{aligned}
c_{1} & =\delta \cos \left(\frac{\alpha_{0}}{2}\right) \cos \left(\frac{\beta}{2}\right), \\
c_{2} & =\cos \frac{\alpha_{0}}{2}\left(\delta \cos \alpha_{0} \sin \frac{\beta}{2}+\sin \alpha_{0} \sqrt{\Delta^{2}-\delta^{2}}\right), \\
c_{3} & =\sin \frac{\alpha_{0}}{2}\left(\Delta-\delta \sin \alpha_{0} \sin \frac{\beta}{2}+\cos \alpha_{0} \sqrt{\Delta^{2}-\delta^{2}}\right) .
\end{aligned}
$$

This kinematic calculation leaves three geometric parameters which govern the deformation of the bending crease model: $\delta, \Delta, \alpha_{0}$.

\section{APPENDIX B: TRANSFORMATION FROM MODEL TO EXPERIMENTAL DISPLACEMENT}

Due to size of the indenter, and the presence of the hole, the disks are not loaded at a central vertex as assumed by the analytical models. Instead, the indenter contacts the creases some distance from vertex. Additionally, the analytical models assume that the disk is supported where the creases reach the edge of the disk; however, for the tests the supports are located closer to the center of the disk. Using the geometry of the disk and test apparatus, a conversion is made between the displacement of the vertex obtained from the models and the corresponding experimentally measured displacement of the indenter.
For the straight crease, shown in Fig. 20(a), $\delta$ is the model displacement measuring the distance from the central vertex to where the crease reaches the disk edge. The experimental displacement, $\delta_{1}^{*}$, is measured from the supports to the contact point of the indenter and the creases. Using the geometry of the test, the relationship between the experimental and model displacements is

$$
\begin{aligned}
\delta_{1}^{*} & =\delta-\frac{d_{i}}{2}\left(\frac{\delta}{R}\right)-\frac{\delta}{R}\left[R-\frac{L}{2} \sqrt{1-\left(\frac{\delta}{R}\right)^{2}}\right] \\
& \approx \frac{\delta\left(L-d_{i}\right)}{2 R} .
\end{aligned}
$$

For the bending crease a dimple of radius $\Delta$, measured along the crease, forms and deformation primarily occurs within this dimple; see Fig. 20(b). Similar to the straight crease, $\delta$ is the model displacement coordinate measuring the distance from the central vertex to the dimple edge. The experimental displacement, $\delta_{1}^{*}$, is measured from the supports to the contact point of the indenter and the creases. Using the geometry of this configuration, the relationship between experimental and model displacements found:

$$
\delta_{2}^{*}=\delta-\frac{d_{i}}{2}\left(\frac{\delta}{\Delta}\right)=\delta\left(1-\frac{d_{i}}{2 \Delta}\right)
$$

When the number of creases is greater than two, the creases do not initially lie in the same plane as the supports. As a result, the experimental displacement $\delta_{3}^{*}$ is measured from the shape in the lowest energy stable state, as shown in Fig. 21. The model displacement, $\delta$, is measured from the crease edge to the central vertex. When the deformation of the disk raises the central vertex above the supports $(\delta<0)$, the contact between the indenter and the disk is at the hole edge, therefore

$$
\delta_{3}^{*}=\left(\frac{L}{2} \tan \Psi-r \sin \Psi\right)-\left(\frac{L}{2} \tan \Psi_{0}-r \sin \Psi_{0}\right),
$$

where the first bracketed term is the distance from the support to the edge of the hole in the deformed state and the second bracketed term is the distance from the support to the hole edge in the natural state. Noting that $\sin \Psi=\delta / R$ and $\Psi_{0}$ is the crease inclination in the natural state,

$$
\begin{aligned}
\left(\delta_{3}^{*}\right)_{\delta<0} & =\left(\frac{L}{\sqrt{1-\frac{\delta^{2}}{R^{2}}}}-2 r\right) \frac{\delta}{2 R}-\frac{L}{2} \tan \Psi_{0}+r \sin \Psi_{0} \\
& \approx(L-2 r) \frac{\delta}{2 R}-\frac{L}{2} \tan \Psi_{0}+r \sin \Psi_{0} .
\end{aligned}
$$

TABLE II. Natural state crease inclinations obtained from Ref. [14].

\begin{tabular}{ccccc}
\hline \hline$N$ & 2 & 3 & 4 & 5 \\
\hline$\Psi_{0}$ & 0 & $-0.12 \beta$ & $-0.17 \beta$ & $-0.19 \beta$ \\
\hline \hline
\end{tabular}


When the creases pass through the plane of the supports $(\delta>0)$ the contact point switches to the indenter edge, as shown in Fig. 20:

$$
\left(\delta_{3}^{*}\right)_{\delta>0} \approx\left(L-d_{i}\right) \frac{\delta}{2 R}-\frac{L}{2} \tan \Psi_{0}+r \sin \Psi_{0}
$$

Noting the first term in Eq. (B4) is equal to $\delta_{1}^{*}$, by analogy for the bending crease model,

$$
\delta_{4}^{*}=r \sin \Psi_{0}-\frac{L}{2} \tan \Psi_{0}+ \begin{cases}\left(1-\frac{r}{\Delta}\right) \delta & \text { if } \delta<0, \\ \left(1-\frac{d_{i}}{2 \Delta}\right) \delta & \text { if } \delta>0 .\end{cases}
$$

From Lechenault and Adda-Bedia [14] the crease inclination in the natural states are given in Table II.
[1] L. Wilson, S. Pellegrino, and R. Danner, in Proceedings of the 54th AIAA/ASME/ASCE/AHS/ASC Structures, Structural Dynamics, and Materials Conference (AIAA, Boston, MA, 2013).

[2] D. S. De Focatiis and S. D. Guest, Philos. Trans. R. Soc. A 360, 227 (2002).

[3] J. L. Silverberg, A. A. Evans, L. McLeod, R. C. Hayward, T. Hull, C. D. Santangelo, and I. Cohen, Science 345, 647 (2014).

[4] M. Schenk and S. Guest, Proc. Natl. Acad. Sci. U.S.A. 110, 3276 (2013).

[5] Z. Abel, J. Cantarella, E. D. Demaine, D. Eppstein, T. C. Hull, J. S. Ku, R. J. Lang, and T. Tachi, J. Comput. Geometry 7, 171 (2016).

[6] K. Liu and G. H. Paulino, Proc. R. Soc. A 473, 20170348 (2017).

[7] J. M. Gattas and Z. You, Int. J. Impact Eng. 73, 15 (2014).

[8] S. W. Grey, F. Scarpa, and M. Schenk, Phys. Rev. Lett. 123, 025501 (2019).

[9] A. A. Evans, J. L. Silverberg, and C. D. Santangelo, Phys. Rev. E 92, 013205 (2015).

[10] M. Schenk and S. Guest, Origami 5: Fifth International Meeting of Origami Science, Mathematics, and Education (5OSME), edited by P. Wang-Iverson, R. J. Lang, and M. Yim (CRC, Boca Raton, FL, 2011), p. 291.

[11] Z. Y. Wei, Z. V. Guo, L. Dudte, H. Y. Liang, and L. Mahadevan, Phys. Rev. Lett. 110, 215501 (2013).

[12] E. T. Filipov, K. Liu, T. Tachi, M. Schenk, and G. H. Paulino, Int. J. Solids Struct. 124, 26 (2017).
[13] F. Lechenault, B. Thiria, and M. Adda-Bedia, Phys. Rev. Lett. 112, 244301 (2014).

[14] F. Lechenault and M. Adda-Bedia, Phys. Rev. Lett. 115, 235501 (2015).

[15] I. Andrade-Silva, M. Adda-Bedia, and M. A. Dias, Phys. Rev. E 100, 033003 (2019).

[16] M. G. Walker and K. A. Seffen, Thin-Walled Struct. 124, 538 (2018).

[17] A. Vaziri, Thin-Walled Struct. 47, 692 (2009).

[18] E. Cerda and L. Mahadevan, Phys. Rev. Lett. 80, 2358 (1998).

[19] T. Liang and T. A. T. Witten, Phys. Rev. E 71, 016612 (2005).

[20] E. Cerda, S. Chaieb, F. Melo, and L. Mahadevan, Nature 401, 46 (1999).

[21] L.D. Landau, E.M. Lifshitz, S.P. Timoshenko, and J. N. Goodier. Theory of Elasticity, 3rd ed. (McGraw-Hill, London, 1970).

[22] The Mathworks Inc., "MATLAB R2018a” (2018).

[23] C. Calladine, Theory of Shell Structures (Cambridge University Press, Cambridge, 1983).

[24] K. A. Seffen, Phys. Rev. E 97, 023004 (2018).

[25] J. P. Duncan and J. L. Duncan, Proc. R. Soc. A 383, 191 (1982).

[26] A. Lobkovsky, S. Gentges, H. Li, D. Morse, and T. A. Witten, Science 270, 1482 (1995).

[27] A. E. Lobkovsky, Phys. Rev. E 53, 3750 (1996).

[28] A. Boudaoud, P. Patrício, Y. Couder, and M. B. Amar, Nature 407, 718 (2000).

[29] D. Hilbert and S. Cohn-Vossen, Geometry and the Imagination, 2nd ed. (Chelsea, New York, 1990). 